\title{
ArcheoSciences
}

Revue d'archéométrie

40 | 2016

Varia

\section{Le complexe minier gaulois des Barrencs (Aude, France) dans son contexte géologique et minéralogique}

The Barrencs Gallic mining network (Aude, France) in its geological and mineralogical context

Gabriel Munteanu, Eric Kammenthaler, Julien Mantenant, Christian Rico, Jean-Marc Fabre et Argitxu Beyrie

\section{OpenEdition}

Journals

Édition électronique

URL : https://journals.openedition.org/archeosciences/4839

DOI : 10.4000/archeosciences.4839

ISBN : 978-2-7535-5384-2

ISSN : 2104-3728

Éditeur

Presses universitaires de Rennes

Édition imprimée

Date de publication : 30 décembre 2016

Pagination : 163-180

ISBN : 978-2-7535-5382-8

ISSN : 1960-1360

Référence électronique

Gabriel Munteanu, Eric Kammenthaler, Julien Mantenant, Christian Rico, Jean-Marc Fabre et Argitxu Beyrie, « Le complexe minier gaulois des Barrencs (Aude, France) dans son contexte géologique et minéralogique », ArcheoSciences [En ligne], 40 | 2016, mis en ligne le 30 décembre 2018, consulté le 23 février 2022. URL : http://journals.openedition.org/archeosciences/4839; DOI : https://doi.org/ 10.4000/archeosciences.4839 


\title{
Le complexe minier gaulois des Barrencs (Aude, France) dans son contexte géologique et minéralogique
}

\author{
The Barrencs Gallic Mining Network (Aude, France) \\ in its Geological and Mineralogical Context
}

\author{
Gabriel Munteand ${ }^{\mathrm{a}}$, Eric Kammenthaler ${ }^{\mathrm{b}}$, Julien Mantenant ${ }^{\mathrm{c}}$, Christian Rico ${ }^{\mathrm{a}}$, \\ Jean-Marc Fabre et Argitxu BeYrie ${ }^{\mathrm{b}}$
}

\begin{abstract}
Résumé : Les travaux miniers anciens des Barrencs, situés dans la vallée de l'Orbiel sur un plateau qui sépare les villages de Lastours et de Fournes-Cabardès (Aude), ont été découverts dès la fin du XIX siècle. Pendant plus d'un siècle ils ont été présentés comme l'œuvre des Romains. Entre 2009 et 2012 les travaux miniers ont fait l'objet d'un projet de recherche porté par une équipe spécialisée dans l'archéologie minière du laboratoire TRACES de l'université de Toulouse 2 - Jean-Jaurès. Les recherches ont mis en évidence un vaste complexe minier qui a exploité des structures filoniennes verticales à sub-verticales démontrant une parfaite organisation et un haut niveau technique de la part des mineurs. Cette nouvelle étude a permis de faire remonter la plus grande partie de l'activité minière au second âge du Fer. L'étude géologique menée en interaction directe avec les investigations archéologiques démontre une excellente maîtrise de l'exploitation, basée sur une bonne connaissance géologique empirique. Elle a guidé les anciens mineurs lors de la reconnaissance du gisement et, par la suite, pour l'exploitation des zones riches. Les analyses minéralogiques et élémentaires réalisées sur un échantillonnage systématique conduit au sein des travaux étudiés mettent en évidence une minéralisation épithermale riche en cuivre, argent et plomb. La composition minéralogique des minerais du gisement est dominée par la pyrite, la chalcopyrite, les « cuivres gris » argentifères, la galène et la galène argentifère, la sphalérite et des sulfures de cuivre souvent argentifères. Des sulfures et des sulfosels complexes d'argent, de cuivre et de plomb constituent la fraction mineure. Il ressort que pour les anciens mineurs, le minerai exploité était riche en cuivre, en argent et en plomb. Mais, la mine est loin d'avoir révélé tous ses secrets et des recherches complémentaires seront nécessaires pour estimer les volumes de minerai extrait, les modalités de traitement des minerais, le type de production ou le commerce.
\end{abstract}

\begin{abstract}
The ancient mining works of Barrencs, situated in the Orbiel valley on a plateau between the Lastours and Fournes-Cabardès villages (Aude, France), were discovered at the end of the $19^{\text {th }} \mathrm{c}$. For more than a century these were thought to be Roman in origin. Between 2009 and 2012 , the mining works have been studied as part of a research program carried out by a mining archaeological specialised team of TRACES laboratory of Toulouse 2 Jean-Jaurès University. The study identified a vast mining complex that exploited vertical to sub-vertical vein structures, showing perfect organisation and a high technical level of the miners. It also permitted to push back in time the mining activity, to the Iron Age. The geological study, carried out in close relation with the archaeological investigations, shows an excellent mining proficiency, based on a good geologic empirical knowledge that guided the ancient miners, both for ore deposit survey and for its eventual extraction. The mineralogical and elemental analyses conducted via a systematic sampling through out the entire studied mine area, showed that the area under study is rich in copper, silver, and lead epithermal mineralisations. The composition of mineralogical ore deposits is dominated by pyrite, chalcopyrite, silver-bearing "gray coppers", galena and silver-bearing galena, sphalerite, and copper sulphides (often argentiferous). Complex silver-copper-lead sulphides and thiosalts are present in smaller proportions. It appears that, for the ancient miners, the mined ore was rich in copper, silver and lead. Theses first results are quite promising and further study will focus on other issues, such as the ore volumes extracted, the ore treatment methods, and the types of production or trading areas; all important for the total comprehension of this ancient mining complex.
\end{abstract}

a Université de Toulouse 2-Jean Jaurès, TRACES-UMR 5608 CNRS, 5 allée Antonio-Machado, 31058 ToULOUSE. (gbmunt@gmail.com) (rico@univ-
tlse2.fr) (jfabre@univ-tlse2.fr)
'IKER Archéologie et Patrimoine, Pelosastrea,64780 SAINT-MARTIN-D'ARROSSA, membre associé CNRS-TRACES-UMR 5608. (eric.kammenthaler@
iker-archeologie.com) (argitxu.beyrie@iker-archeologie.com)
'ACTERArchéologie, 1 rue du Marché-de-Gros, 66370 PÉZILLA-LA-RIVIÈRE, membre associé CNRS-TRACES-UMR5608. (mantenantjulien@yahoo.fr) 
Mots clés : âge du Fer, argent, cuivre, gîtologie, mine, plomb.

Keywords: Iron Age, silver, copper, ore deposit, mine, lead.

\section{INTRODUCTION}

\section{Localisation et historique des recherches}

À seulement quelques kilomètres au nord de Carcassonne, dans les terrains appartenant aux contreforts sud de la Montagne Noire, se situe le riche district polymétallique de Salsigne (figure $1 \mathrm{~b}$ ), notamment très connu pour l'exploitation de l'or et de l'arsenic tout au long du siècle passé Précédemment jusqu'à la fin du XIx ${ }^{\mathrm{e}}$ siècle, moment de la découverte de l'or, l'intérêt des exploitants miniers s'était porté sur d'autres métaux, notamment sur le fer, l'argent, le cuivre et le plomb. Pour l'époque antique, de nombreux centres sidérurgiques sont aujourd'hui bien connus pour la production du fer tiré de " chapeaux de fer " situés sur le versant méridional de la Montagne Noire $(20 \mathrm{~km}$ au nord de Salsigne) et formés au-dessus des gisements primaires de pyrite et d'arsénopyrite (Domergue et al., 1993; Decombeix et al., 2001; en dernier lieu, Fabre, Domergue et Dabosi [dir.], 2016).

En 1893, M. Esparseil ${ }^{1}$, « l'inventeur » de l'or à Salsigne, effectue des recherches minières dans le bassin de l'Orbiel, sur le petit plateau des Barrencs ${ }^{2}$, entre Lastours et FournesCabardès. Il découvre et déblaye d'anciennes fosses le long de deux groupes filoniens verticaux : au Mourral de la Grave et $150 \mathrm{~m}$ à l'est des Barrencs des Fournes (Esparseil, 1893). En raison de la multitude de débris d'amphores retrouvés au sein et aux alentours de ces amples et profonds travaux miniers, il les attribue à l'époque romaine. Les recherches minières sur le gisement des Barrencs se poursuivent en plusieurs épisodes jusqu'aux années 1960. Elles ont conduit à l'ouverture de plus de $600 \mathrm{~m}$ de galeries qui ont systématiquement recoupé en profondeur d'anciens chantiers d'exploitation (figure 1a). Ces travaux n'ont jamais débouché sur une réelle phase d'exploitation, les Anciens ayant épuisé la quasi totalité du gisement. Ces recherches minières ont eu le mérite de révéler un site minier ancien, bien conservé, exceptionnel par ses dimensions ${ }^{3}$ (figure 1c) et la qualité de ses ouvrages. Les travaux modernes ont par ailleurs permis

1. M. Esparseil était un ingénieur des mines et géologue.

2. En occitan " barrenc » signifie trou, ravin.

3. Les vestiges miniers se développent sur une superficie de $800 \mathrm{~m}$ de longueur et $350 \mathrm{~m}$ de largeur et sur $200 \mathrm{~m}$ de profondeur minimum. le démarrage de l'étude gîtologique et minéralogique du gisement.

Au cours des années 1970, le site est fermé et, malgré son immense potentiel archéologique, il reste oublié jusqu'aux années 1990 quand quelques brèves explorations sont réalisées par les représentants des services de l'État (DIREN, DRAC-SRA) ${ }^{4}$. En 2001, D. Morin ${ }^{5}$ a fait une étude exploratoire sur les travaux miniers des Barrencs de Fournes situés à l'est des travaux du Mourral de la Grave (Morin, 2001). Cependant, il a fallu attendre 2009 pour qu'une partie du Mourral de la Grave fasse l'objet d'une étude complète d'archéologie minière par des spécialistes ${ }^{6}$ dans le cadre d'un programme de recherche achevé en $2012^{7}$.

\section{Les Barrencs, une vaste mine du second âge du Fer à l'Antiquité}

Comme mentionné précédemment, le gisement des Barrencs est constitué par deux structures filoniennes Mourral de la Grave et Barrencs de Fournes (figure 1a). Situé sur la bordure Est du plateau, l'ensemble de Barrencs de Fournes regroupe de grands chantiers à ciel ouvert qui ont exploité en surface trois filons, deux de direction N-S et un filon croiseur de direction NO-SE. Mais ce dernier filon n'a fait l'objet que d'une investigation exploratoire. Nos recherches de 2009 à 2012 se sont concentrées sur les travaux du Mourral de la Grave.

À ce jour les travaux souterrains reconnus sur la structure filonienne du Mourral de la Grave et accessibles seulement

4. Le dossier "Barrencs " a fait l'objet d'un suivi de la part de Ph. Galant, chargé de la gestion des grottes ornées et du domaine souterrain au Service Régional de l'Archéologie Languedoc-Roussillon et P. Cabrol (DIREN).

5. À la demande de la DIREN Languedoc-Roussillon, une expertise archéologique a été réalisée en octobre 2001 par D. Morin (enseignantchercheur du Laboratoire TRACES) sur les travaux « des Barrencs de Fournes".

6. Les auteurs (l'équipe archéologique du laboratoire TRACES de Toulouse) remercient à cette occasion les collaborateurs à l'étude : M. Llubes (UPS-GET), N. Morrel i Cortes (UTJJ-TRACES) et L. Arboledas (UTJJ-TRACES).

7. Le programme de recherche a été financé par le Ministère de la Culture, la région Languedoc-Roussillon et la commune de Lastours. À l'issue de cette recherche, une série d'articles ont été publiés et sont intégrés dans la partie bibliographique de cet article. 


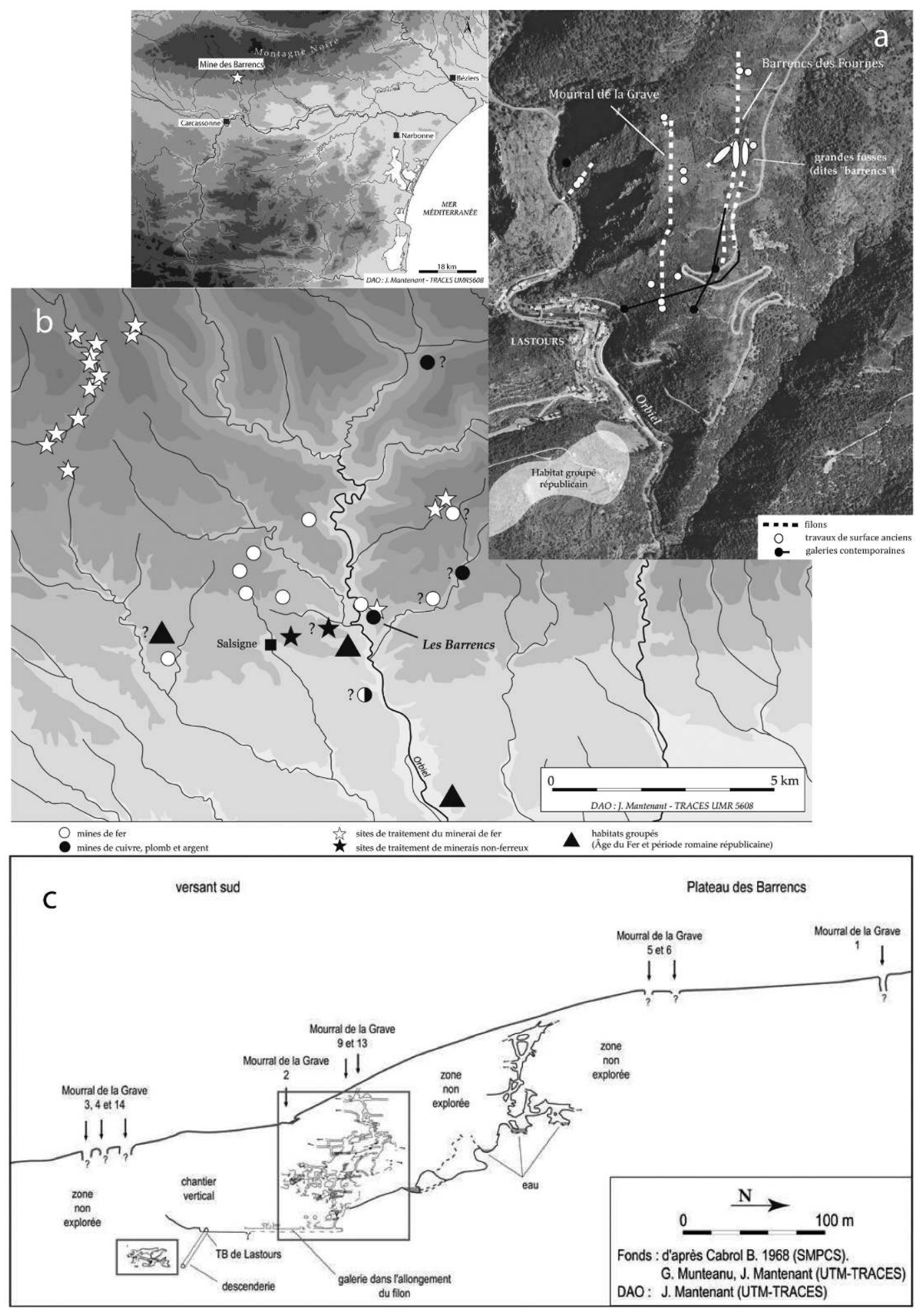

Figure $1: \mathrm{a}$ - Localisation des structures filoniennes et des principaux vestiges miniers de surface du plateau des Barrencs; $b$ - Le district de Salsigne avec la localisation des principaux sites miniers et métallurgiques préindustriels; c - Coupe d'ensemble du réseau du Mourral de la Grave - état actuel des connaissances. Figure 1: $a$-Localisation of vein structures and main mining surface remnants on Barrencs plateau; $b-$ The Salsigne mining district and position of principal preindustrial mining and metallurgical sites; $c$ - General longitudinal-cut through the Mourral de la Grave network - current state of knowledge. 
par le travers-banc moderne de Lastours (percé à partir de 1911), sont impressionnants. Lors de son exploitation, la mine était desservie par de nombreuses ouvertures en surface, situées tout le long de l'affleurement du gisement. Toutes ces entrées sont actuellement comblées et cachées par la végétation abondante et seuls les vestiges de neuf d'entre elles ont pu être repérés (Mourral 1, 2, 3, 4, 5, 6, 9, 13 et 14, voir figures 1a et c). Caractérisés par une verticalité accentuée, les travaux souterrains (figure 1c) se développent sur $320 \mathrm{~m}$ dans l'allongement filonien et, en profondeur, entre le sommet du plateau des Barrencs (380 m d'altitude) et jusqu'à $195 \mathrm{~m}$ d'altitude minimum (cote du niveau hydrostatique où les travaux sont complètement noyés et donc difficiles à explorer). L'étude menée entre 2009 et 2012 a concerné seulement la partie Sud accessible du réseau ancien du Mourral de la Grave (figure 2a). Ce secteur Sud reconnu sur $200 \mathrm{~m}$ de longueur entre 325 et $195 \mathrm{~m}$ d'altitude couvre une surface globale de $25000 \mathrm{~m}^{2}$, dont environ $15000 \mathrm{~m}^{2}$ d'ouvrage miniers. Ces travaux se présentent comme une succession de chantiers verticaux à sub-verticaux pouvant atteindre 4 à $5 \mathrm{~m}$ de largeur, reliés par un réseau de puits et de galeries. En fonction de la dureté de la roche, deux techniques d'abattage ont été mises en œuvre, à la pointerolle ou par le feu, mais l'usage mixte feu/pointerolle a été observé à plusieurs endroits. Si le travail à l'outil a été employé plutôt en roche friable ou moyennement dure, comme il a été constaté dans la partie sud des travaux étudiés, encaissés dans des schistes et quasiment dépourvus de l'abattage par le feu, l'usage du feu a été de plus en plus fréquent dans l'allongement septentrional du gisement.

Une des questions posées lors de l'étude était de déterminer la chronologie de l'activité de ce vaste complexe minier. Jusqu'en 2009, le site était considéré d'époque romaine républicaine (fin du $\mathrm{II}^{\mathrm{e}}$-première moitié du $\mathrm{I}^{\text {er }}$ siècle av. $\mathrm{n}$. è.), d'après les vestiges céramiques éparpillés autour des travaux en surface, sur le plateau et ses versants : fragments de lampes campaniennes, campaniennes A, communes romaines et amphores italiques Dressel 1 . Or, le doute est venu avec une datation ${ }^{14} \mathrm{C}$ par P. Cabrol en 1996 sur un fragment de charbon de bois provenant de la partie Nord des travaux souterrains du Mourral de la Grave. La mesure a donné la date calibrée de 390-200 av. n. è (P. Galant, 2001). L'hypothèse que les travaux étaient exclusivement d'époque romaine dans le contexte de la province Narbonnaise a été remise en question par la suite des découvertes. En effet, la plupart des datations ${ }^{14} \mathrm{C}$ (au total 15 dates) effectuées sur des charbons de bois prélevés en stratigraphie lors des fouilles réalisées par notre équipe entre 2010 et 2011 dans les travaux du Mourral de la Grave (Mantenant et al., 2013) se sont avérées antérieures (figure 2b). Néanmoins, une fréquentation tardo-républicaine ne peut être écartée, car les fourchettes de probabilité de six datations dépassent la charnière des $\mathrm{II}^{\mathrm{e}}-\mathrm{I}^{\mathrm{er}}$ siècles av. n. è. et de nombreux fragments de Campanienne A, d'amphores vinaires gréco-italiques et Dressel 1 , de céramiques de tradition gauloise mais aussi ibérique (sombrero de copa) sont dispersées dans les zones supérieures des travaux souterrains. Cependant, il est à signaler aussi qu'aucun fragment de céramique n'a été retrouvé enfoui dans un comblement minier en place. Dans le meilleur des cas, les céramiques rencontrées en souterrain se trouvaient positionnées dans la couche supérieure des séquences stratigraphiques des sondages archéologiques. Par ailleurs, en surface et tout autour des accès au réseau souterrain (puits, chantiers à ciel ouvert et galeries), se rencontre en grand nombre le même type de mobilier céramique varié, arrivé là, semble-t-il, dans un contexte de décharge domestique ${ }^{8}$ ou de remaniement des niveaux d'occupation antique, notamment par les travaux agricoles postérieurs. À l'avenir, d'autres sondages dans le remplissage minier contemporain de l'exploitation seront nécessaires pour mieux éclairer cette présence tardo-républicaine au sein des vestiges miniers. Pour l'heure, les données en présence montrent une intervention d'époque romaine sur le site qui serait plutôt d'ordre exploratoire, voire de recherche, non une véritable exploitation. Aussi, l'activité minière principale se situe-t-elle essentiellement dans les périodes de La Tène ancienne et moyenne.

\section{Objectifs et méthodologie}

Dans ce programme, concentré sur une partie des travaux du groupe filonien du Mourral de la Grave, les principaux champs de recherche ont été au nombre de trois. D'une part, il s'agissait de mieux cerner la chronologie de l'activité minière, jusque-là dominée par l'hypothèse d'une exploitation romaine. D'autre part, il était nécessaire de mener une étude détaillée sur l'organisation et les techniques mises en œuvre pour réaliser une exploitation minière d'une telle envergure. Enfin, la dernière étude s'est concentrée sur une approche pétrographique, géo-structurale, gîtologique et minéralogique du site en complément des recherches géologiques antérieures. Les principaux objectifs étaient d'identifier dans le détail les types de minéralisations exploitées et les métaux potentiellement extraits par les Anciens et de contribuer à mieux comprendre la gestion, la dynamique et

8. À ce jour, nos prospections menées en surface aux abords des travaux miniers n'ont pas pu mettre encore en évidence des niveaux d'occupation en lien avec ces céramiques. 
Figure 2 (Voir planche couleur XXI) : a - Coupe projetée des travaux miniers étudiés du Mourral de la Grave - localisation des sondages archéologiques et des trois exemples de sections de galerie; $b$ - Diagramme des intervalles de datations ${ }^{14} \mathrm{C}$ sur des charbons de bois issus du contexte stratigraphique des sondages archéologiques; c Restitution de la dynamique d'exploitation du secteur Nord des Travaux supérieurs - les couleurs correspondent à différents ensembles et/ou phases d'exploitation; $\mathrm{d}$ - Exemples de sections de galeries de recherche où les mineurs ont utilisé les failles pour creuser et se diriger.

Figure 2 (See colour plate XXI): a - Projected longitudinal-cut of Mourral de la Grave studied mining works; $b$ - bar-graph of ${ }^{14} \mathrm{C}$ dating intervals conducted on charcoal pieces recovered in stratigraphic context within test pits; $c$ - reproduction of the north Upper Workings mining dynamics - the different colours correspond to different operating assemblages and/ or stages; $d$ - exploration gallery cross-section examples, where the miners used faults for orientation and excavation.

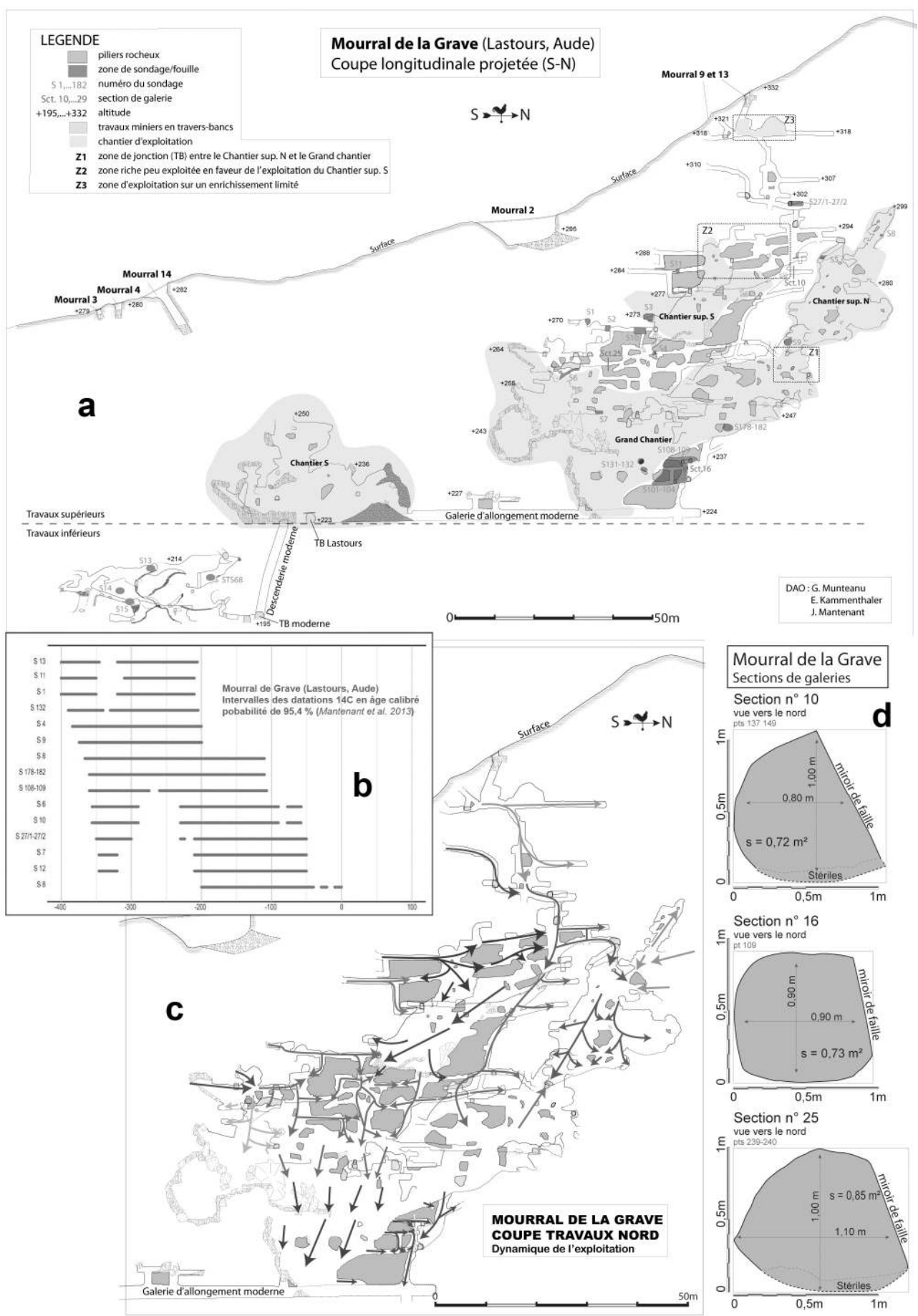

l'architecture de l'espace minier révélées par les investigations archéologiques.

Selon une méthode qui a fait ses preuves sur d'autres mines anciennes en France et en Roumanie9, l'étude des

9. Sites miniers où nous avons participé à l'étude géologique détaillée des travaux miniers, en appui aux investigations archéologiques : Les mines antiques d'or et d'argent de Rosia Montana, Roumanie (recherche parements des ouvrages miniers a abouti à l'élaboration d'une cartographie géologique. Sur cette carte sont reportées toutes les observations et analyses relatives aux limites et à la

coordonnée par B. Cauuet, TRACES-UMR5608); Les mines d'argent du Fournel, Argentière-la-Bessée (recherche coordonnée par B. Ancel, CCSTI-Argentière-La-Bessée); Le district minier de Baillestavy, Pyrénées Orientales (recherche coordonnée par C. Tamas, G. Munteanu et G. Mut - TRACES-UMR5608). 
typologie des corps de minerai, aux accidents géologiques (failles, fissures, décrochements), à la nature du minerai et de la roche encaissante (texture, structure, teneur et composition minéralogique). Dans cette perspective, il a été fait un échantillonnage systématique de la roche et du minerai dans les zones remarquables (54 échantillons) de tout l'espace minier étudié (figure 3a). Après une caractérisation macroscopique, tous les échantillons de minerai (46 échantillons) ont fait l'objet d'analyses chimiques élémentaires (analyses effectuées par ALS Global ${ }^{10}$ ) pour déterminer les teneurs, notamment en métaux susceptibles d'intéresser les Anciens (tableau 1). Une série de 25 échantillons ont été préparés (lames polies et lames minces) pour une première analyse microscopique optique ${ }^{11}$, afin de déterminer les minéraux porteurs des métaux et les minéraux associés. Pour mieux caractériser les minéraux métalliques et leurs associations, 10 de ces sections ont été sélectionnées pour être analysées au microscope électronique à balayage (JeolJSM 6360 LV) et sept ont fait l'objet d'analyses quantitatives à la microsonde électronique ${ }^{12}$ (synthèse des résultats présentée tableaux 4 et 5).

\section{ApPorTS DE L'ANALYSE GÉO-ARCHÉOLOGIQUe}

\section{La structure filonienne du Mourral de la Grave}

\section{Contexte géologique général}

Le plateau des Barrencs fait partie des plissements nordminervois des terrains fossilifères datés du Cambrien au Dévonien, inclus dans l'Unité de Salsigne-Fournes (figure 4). Le soubassement est constitué par les Schistes X au-dessus desquels les nappes de charriage mises en place lors de l'orogénie hercynienne se déversent en direction du sud. Daté du Cambrien inférieur au Viséen, le faciès rocheux de l'encaissant des minéralisations présente une variation en bandes qui se succèdent ainsi du nord au sud : calcaires et dolomies $(150 \mathrm{~m})$, alternance de calcaires blancs et schistes $(50 \mathrm{~m})$, schistes $(40 \mathrm{~m})$, calcaires dolomitiques $(80 \mathrm{~m})$ (Issard, 1984). La pointe Sud du plateau est constituée par la formation siliceuse des Grès de Marcory.

Les structures filoniennes repérées sur 500 à $600 \mathrm{~m}$ de longueur et groupées en deux ensembles qui bordent à l'est et à l'ouest le plateau (Mourral de la Grave et Barrencs des

10. Pour plus de détails sur les types et méthodes d'analyses voir le site [www.alsglobals.com].

11. Microscope optique Zeiss Axioimager A2 m doté d'une caméra numérique Zeiss Axiocam I5 pilotée par le logiciel Zeiss Axiovision.

12. Microsonde CAMECA SX50, tension $25 \mathrm{kV}$, intensité $20 \mathrm{nA}$ et faisceau de $\phi=9 \mu \mathrm{m}^{2}$.
Fournes), sont conditionnées tectoniquement dans le cadre régional du système de failles cassantes majeures orientées N-S (Yueshi Lei, 1993). Des failles de direction NO-SE repérées sur le plateau croisent les premières (figure 1a). Les deux poussées hydrothermales de basse température (épithermales) successives sont reliées à la tectonique cassante tardi-hercynienne; elles se produisirent très probablement au Carbonifère supérieur. Les fluides minéralisateurs ont remonté les fractures de l'encaissant déposant, au fur et à mesure de leur dépressurisation et refroidissement, les minéralisations, notamment métallifères. Dans leur partie supérieure, les filons sont verticaux à sub-verticaux et d'une puissance qui ne dépasse pas $1,5 \mathrm{~m}$ (Crouzet et Tollon, 1980). En profondeur, le contexte tectonique apporte des variations plus complexes, principalement induites par le mouvement général de décrochement sénestre, suivi d'une distension des failles principales, avec une influence directe sur la puissance et la richesse des corps minéralisés.

En interaction directe avec l'étude archéologique, nos investigations sur le conditionnement tectonique, la morphologie et la distribution des corps minéralisés, ainsi que leurs relations avec l'encaissant, ont principalement porté sur les travaux souterrains. Ces derniers concernent principalement la partie centrale de la structure filonienne du Mourral de la Grave, séparés arbitrairement ${ }^{13}$ par notre équipe en Travaux supérieurs et Travaux inférieurs.

\section{Le contrôle tectonique de la minéralisation}

Les Travaux supérieurs se sont développés dans un contexte gîtologique comprenant trois panneaux filoniens principaux (F1, F2 et F3) qui constituent le remplissage hydrothermal mis en place lors d'une phase de distension des failles orientées principalement N-S (figure 3a). Si les filons $\mathrm{F} 1$ et $\mathrm{F} 2$ sont verticaux à sub-verticaux (pendage Est F1 et Ouest F3 entre $70^{\circ}$ et $85^{\circ}$ ), le filon F2, recoupé par les deux précédents, est incliné (pendage Est entre $55^{\circ}$ et $65^{\circ}$ ). Un quatrième panneau filonien $(\mathrm{F} 4)$ constitue le remplissage d'une faille transversale (dir. E-O, pendage $50^{\circ}$ Sud), bordée par les trois autres. Conjointement aux failles principales, d'autres failles et fissures de moindre envergure sont apparues, disposées parallèlement ou transversalement aux principales. Ces fractures ont constitué le support de branches filoniennes plus ou moins minéralisées et recherchées par les Anciens (figure 3c). D'autres mouvements tectoniques sont survenus après la mise en place des minéralisations. Ceux-ci ont réactivé les failles initiales induisant ainsi l'apparition de nouvelles fractures. Des failles et des

13. Par des considérations de positionnement dans l'espace nous avons choisi comme limite entre les Travaux supérieurs et les Travaux inférieurs le niveau moderne du travers-banc (TB) de Lastours. 
Figure 3 (Voir planche couleur XXII) : a - Coupe gîtologique du secteur étudié de la structure filonienne du Mourral de la Grave avec la localisation des lieux d'échantillonnage et des coupes (b) et du plan (c); b - Coupes transversales (1 et 2) dans la structure filonienne; c-Vue en plan de la structure filonienne.

Figure 3 (See colour plate XXII): a Longitudinal-cut of the ore deposit structure in the studied area of Mourral de la Grave with localised sampling points, cross-cuts sections (b) and plan (c); bCross-cuts (1 and 2) of the vein structure; $c$-plan view of the vein structure.

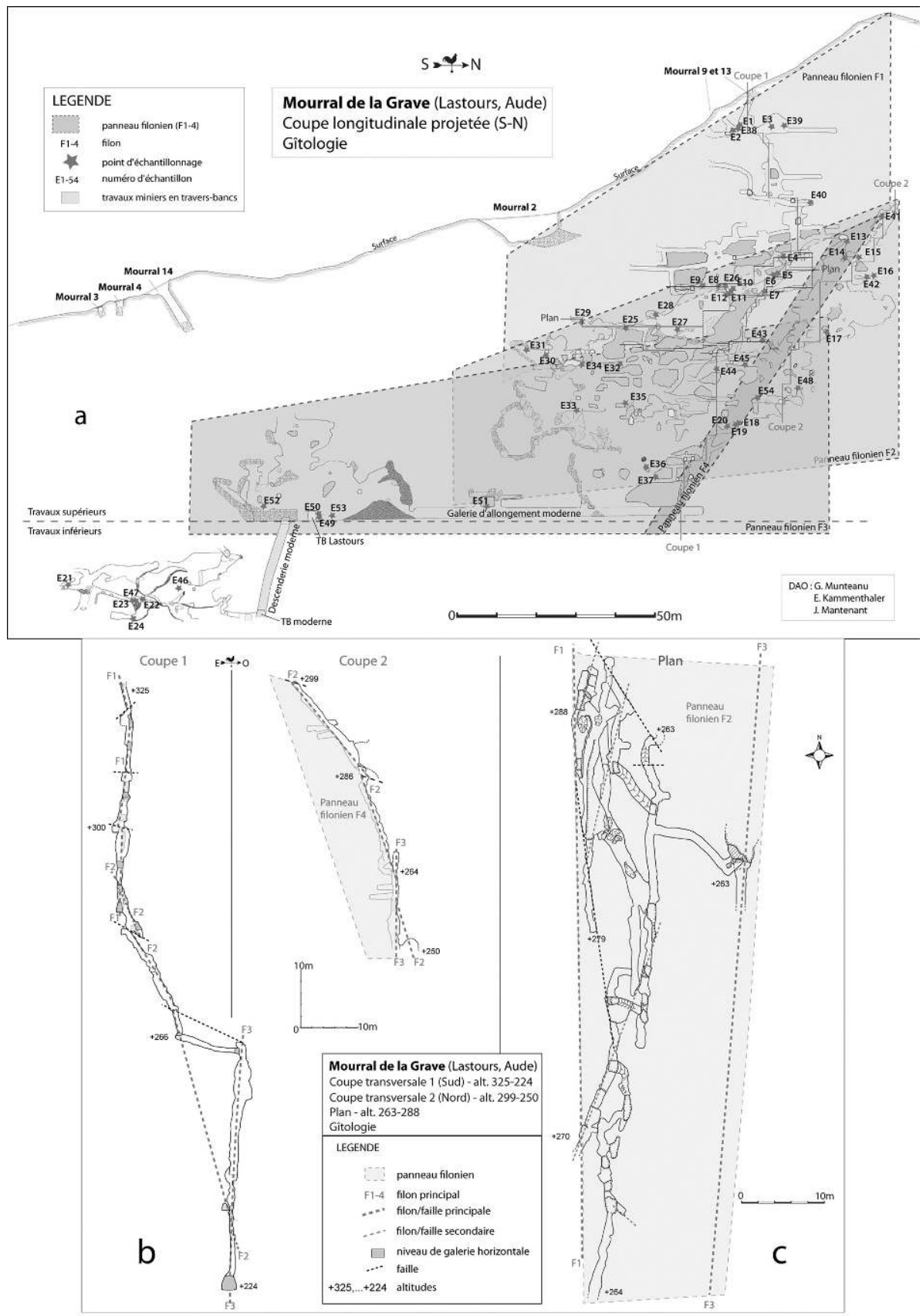

fissures horizontales à subhorizontales, issues notamment de l'activation des contacts lithologiques de l'encaissant, ont cisaillé et fragmenté les filons. Ce phénomène a pu être suivi et relevé notamment sur les filons F1 et F2 (figure 3b). Les mouvements des terrains (litons) ${ }^{14}$ le long des failles et les interactions qui en résultent sont difficiles à observer, car un important phénomène de concrétionnement karstique s'est

14. Un liton est un compartiment rocheux créé par un jeu de failles. instauré depuis la création du vide souterrain et, souvent, des coulées épaisses de calcite recouvrent les traces de friction sur les miroirs de faille.

Les investigations géologiques sur le secteur des Travaux inférieurs ont été plus limitées (figures $2 \mathrm{a}$ et $3 \mathrm{a}$ ), mais nous avons pu remarquer que les minéralisations encaissées généralement dans des calcschistes et des schistes se compliquent un peu dans le plan horizontal. La tendance générale reste la même, dirigée par les failles orientées N-S. 


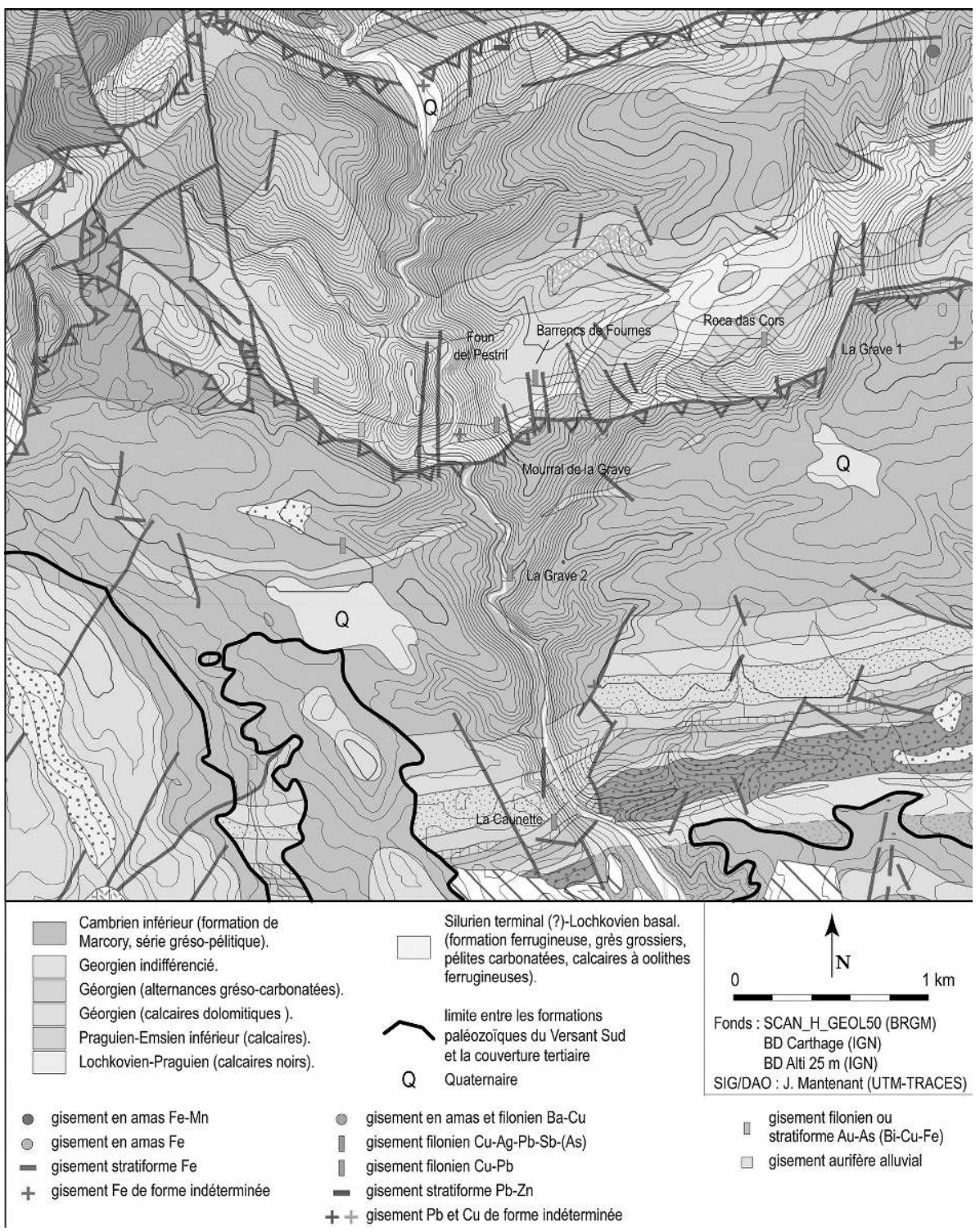

Figure 4 (Voir planche couleur XXIII) : Carte géologique de la région de Lastours.

Figure 4 (See colour plate XXIII): Geological map of Lastours region.

\section{Les textures de minéralisation}

Si à grande échelle les minéralisations ont suivi les failles et les fractures de l'encaissant, le remplissage des vides s'est accompli de plusieurs façons. D'une part, le remplissage des fentes des fractures s'est réalisé en bandes séquentielles, disposées parallèlement, avec un phénomène de diffusion dans les épontes filoniennes plus ou moins conséquent en fonction de la qualité de l'encaissant (figure 5a). D'autre part, dans les zones de l'encaissant de faible cohésion (calcschistes et schistes), le fluide hydrothermal a pénétré profondément dans la masse rocheuse, notamment le long des plans préférentiels de schistosité. Cela a produit une fractu- ration hydraulique en bandes parallèles (figure 5b) et, parfois, la formation d'une structure de type brèche mosaïque (Tamas, 2010) avec un très faible déplacement des clastes. Mais les zones filoniennes les plus puissantes se sont établies au contact et au croisement des failles principales. Par ailleurs, ce sont dans les endroits où les espaces sont plus larges et la fracturation de l'encaissant plus importante que se forment les brèches hydrothermales. Des fragments détachés de l'encaissant, soit par les facteurs tectoniques, soit par la pression créée par le dégazage du fluide hydrothermal, se retrouvent englobés dans le ciment hydrothermal (figure 5c). 
Figure 5 : (Voir planche couleur XXIV) a Remplissage filonien de la fente de la faille directrice du filon F2; b - Structure de minéralisation en bandes, parfois mosaïquée, dans l'encaissant schisteux au toit du F2 ; c - structure de brèche hydrothermale au croisement des filons F2 et F3; d - recherches de minéralisation portée au toit et au mur de la faille du filon F2; e minéralisation massive, à faible participation de la gangue quartzeuse $(\mathrm{Q})$, par une association de freibergite $(\mathrm{Fr})$ dominante, bournonite $(\mathrm{Bn})$, chalcopyrite (Cpy) et galène $(\mathrm{Gn})$ - clichée en microscopie optique sur E54; $\mathrm{f}$ - association de sulfures communes (chalcopyrite, pyrite-Py) avec substitution en zones périphériques et alvéolaires de sulfosels argentiferes (freibergite, gustavite-Gtv) dans une gangue à quartz et ankérite (Ank).

Figure 5: (See colour plate XXIV) a-Ore filling in the F2 lode guide-fault; $b$-striped, even mosaic, mineralisation structure in the F2 lode roof schistose host rock; $c$ - hydrothermal breccias structure at the F2 and F3 junction; $d$-mineralisation survey galleries on the F2 lode wall and roof; $e-$ massive mineralisation with very poor quartz gangue involvement made of a freibergite $(\mathrm{Fr}$ ) dominated association with bournonite (Bn), chalcopyrite (Cpy), and galena (Gn) - optic microscope image on E54; $f$-common sulphides association (chalcopyrite, pyrite-Py) with peripheral and alveolar area substitutions of silver thiosalts (freibergite, gustaviteGtv) within a quartz and ankerite (Ank) gangue.

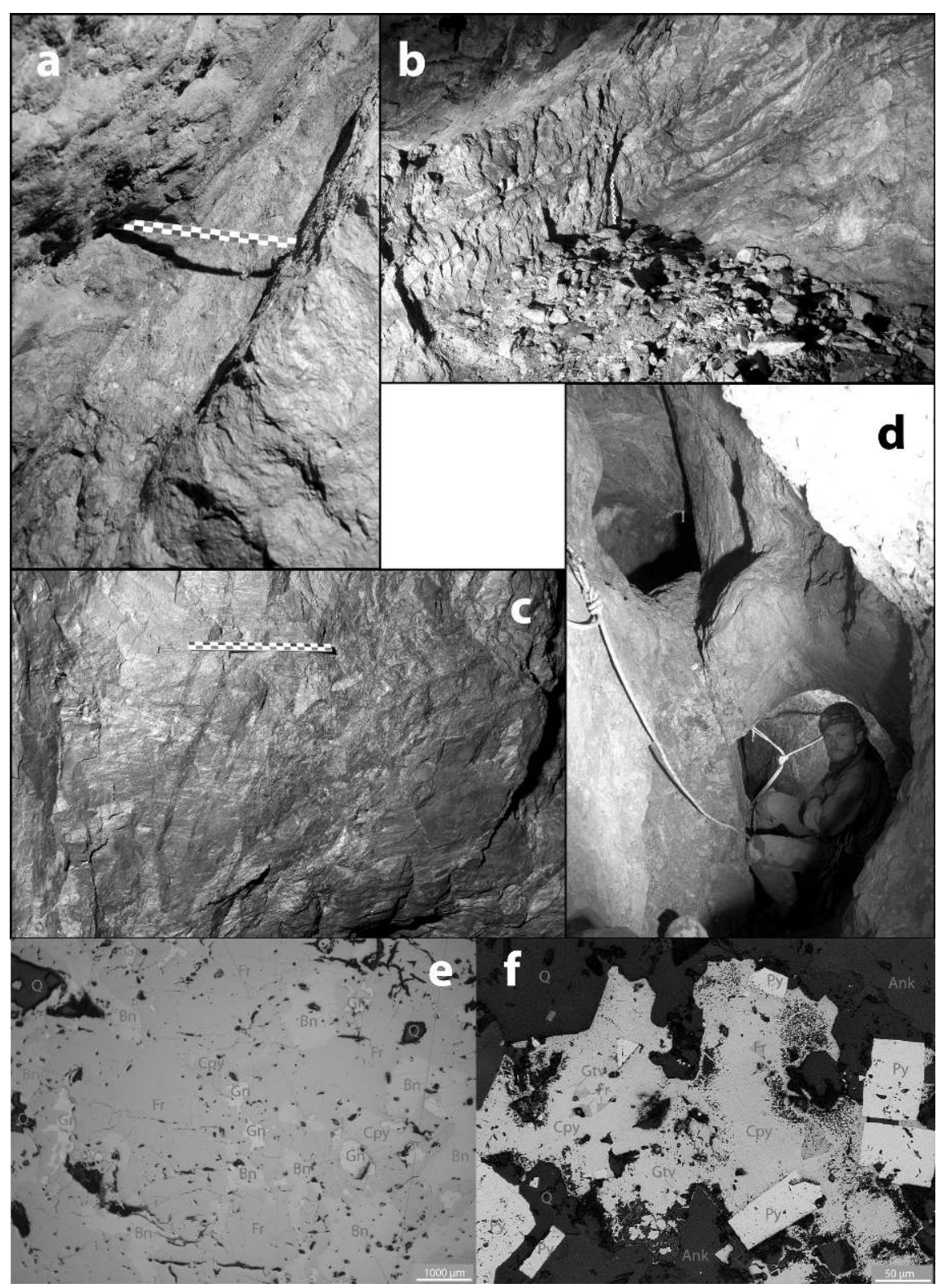

\section{Nature et distribution spatiale de la minéralisation Cu-Ag-Pb du Mourral de la Grave}

\section{Les teneurs en métaux du minerai}

Des analyses géochimiques globales donnant les teneurs élémentaires du minerai ont été effectuées sur la plupart des échantillons prélevés. La synthèse des résultats présentés (tableau 1) est centrée seulement sur les teneurs des métaux susceptibles ${ }^{15}$ d'avoir été exploités aux époques anciennes.

15. Par les textes anciens et par l'archéologie nous savons que les principaux métaux exploités par les Anciens furent : $\mathrm{Au}, \mathrm{Ag}, \mathrm{Fe}, \mathrm{Pb}, \mathrm{Cu}, \mathrm{Sn}$ (Domergue, 2008).
Les échantillons analysés sont constitués de minerai de tout venant (gangue comprise) et les observations en microscopie optique illustrent une participation de la fraction minérale utile (minéraux métalliques) qui peut varier de 10 à $90 \%$ du volume de minerai ${ }^{16}$ (tableau 3 - colonne « \% volume »). Ces pourcentages indiquent clairement qu'en triant et donc en concentrant les parties riches du minerai

16. L'estimation a été réalisée en calculant sur une section polie (obtenue perpendiculairement à la direction majeure des minéralisations) les surfaces des minéraux métalliques par rapport à celle de la gangue, résultats convertis ensuite en $\%$. 


\begin{tabular}{|c|c|c|c|c|c|c|c|c|}
\hline \multicolumn{4}{|c|}{ Méthodes d'analyse } & $\begin{array}{l}\text { AA23 } \\
\text { AA26 }\end{array}$ & $\begin{array}{c}\text { AA46 } \\
\text { AA47 } \\
\text { AA62 } \\
\text { ME-ICP61 } \\
\text { OG-46 }\end{array}$ & $\begin{array}{c}\text { AA47 } \\
\text { ME-ICP61 }\end{array}$ & $\begin{array}{l}\text { ME-ICP41 } \\
\text { ME-ICP61 }\end{array}$ & $\begin{array}{c}\text { AA46 AA47 } \\
\text { ME-ICP61 } \\
\text { OG46 }\end{array}$ \\
\hline \multicolumn{4}{|c|}{ Unité de mesure } & $\mathrm{g} / \mathrm{t}$ & $\mathrm{g} / \mathrm{t}$ & $\mathrm{g} / \mathrm{t}$ & $\mathrm{kg} / \mathrm{t}$ & $\mathrm{g} / \mathrm{t}$ \\
\hline \multirow{2}{*}{ No crt. } & \multirow{2}{*}{ Échantillon } & \multirow{2}{*}{ Provenance } & \multirow{2}{*}{ Poids analysé (g) } & \multicolumn{5}{|c|}{ Métaux potentiellement exploités } \\
\hline & & & & $\mathrm{Au}$ & $\mathrm{Ag}$ & $\mathrm{Cu}$ & $\mathrm{Fe}$ & $\mathrm{Pb}$ \\
\hline 1 & E2 & $\mathrm{F} 1$ & 160 & 0,014 & 10 & 46 & 80,4 & 404 \\
\hline 2 & E3 & F1 & 320 & 0,068 & 53 & 2190 & 122,5 & 1820 \\
\hline 3 & E4 & $\mathrm{F} 1 / \mathrm{F} 2$ & 140 & 0,025 & 30 & 91 & 31,6 & 564 \\
\hline 4 & E6 & $\mathrm{F} 1 / \mathrm{F} 2$ & 460 & 0,055 & 128 & 664 & 41,8 & 76 \\
\hline 5 & E7 & F2 & 660 & $<0.005$ & 6 & 36 & 21,7 & 306 \\
\hline 6 & E8 & F1 & 60 & 0,133 & 19 & 195 & 113 & 342 \\
\hline 7 & E9 & F1 & 140 & 0,02 & 30 & 1545 & 45,6 & 567 \\
\hline 8 & E11 & F2 & 260 & 0,42 & 181 & 2070 & 66,9 & 2180 \\
\hline 9 & E15 & F2 & 100 & 0,059 & 309 & 2710 & 21,5 & 608 \\
\hline 10 & E16 & F2 & 480 & 0,158 & 49 & 332 & 55 & 53 \\
\hline 11 & E17 & F2 & 380 & 0,614 & 169 & 1610 & 54,1 & 20 \\
\hline 12 & E18 & $\mathrm{F} 2 / \mathrm{F} 4$ & 220 & 0,046 & 636 & 3660 & 42,3 & 897 \\
\hline 13 & E19 & $\mathrm{F} 2 / \mathrm{F} 4$ & 180 & 0,011 & 16 & 74 & 23 & 108 \\
\hline 14 & E21 & Tr. Inf. & 260 & 0,344 & 40 & 1090 & 25 & 918 \\
\hline 15 & E22 & Tr. Inf. & 400 & 0,047 & 12 & 1450 & 2,02 & 294 \\
\hline 16 & E23 & Tr. Inf. & 340 & 0,083 & 23 & 102 & 9,7 & 411 \\
\hline 17 & E24 & Tr. Inf. & 240 & $<0.005$ & 2 & 16 & 12,4 & 17 \\
\hline 18 & E26 & $\mathrm{F} 1 / \mathrm{F} 2$ & 260 & 0,066 & 216 & 1220 & 56,5 & 644 \\
\hline 19 & E27 & F2 & 740 & 0,042 & 44 & 702 & 32,8 & 2790 \\
\hline 20 & E28 & $\mathrm{F} 1 / \mathrm{F} 2$ & 360 & 0,23 & 27 & 396 & 0 & 30 \\
\hline 21 & E29 & $\mathrm{F} 1 / \mathrm{F} 2$ & 840 & 0,13 & 0 & 405 & 88,2 & 0 \\
\hline 22 & E30 & F2 & 800 & 0,13 & 0 & 391 & 199 & 0 \\
\hline 23 & E31 & F2 & 1060 & 0,45 & 362 & 6560 & 66,6 & 0 \\
\hline 24 & E32 & F2 & 1320 & 0,02 & 5 & 231 & 13,9 & 314 \\
\hline 25 & E33 & $\mathrm{F} 2 / \mathrm{F} 3$ & 400 & 0 & 12 & 158 & 29,7 & 0 \\
\hline 26 & E34 & $\mathrm{F} 2 / \mathrm{F} 3$ & 820 & 0 & 7 & 168 & 39,4 & 0 \\
\hline 27 & E35 & F2 & 320 & 0,02 & 0 & 74 & 41,6 & 0 \\
\hline 28 & E36 & $\mathrm{F} 2 / \mathrm{F} 3$ & 940 & 0,04 & 63 & 314 & 26 & 2130 \\
\hline 29 & E37 & $\mathrm{F} 2 / \mathrm{F} 3$ & 920 & 0,05 & 490 & 3820 & 27,2 & 23400 \\
\hline 30 & E38 & $\mathrm{F} 1$ & 380 & 0,264 & 239 & 758 & 86 & 501 \\
\hline 31 & E39 & $\mathrm{F} 1$ & 460 & 0,153 & 149 & 427 & 35,9 & 1150 \\
\hline 32 & $\mathrm{E} 40$ & $\mathrm{~F} 1$ & 140 & 0,824 & 61 & 1575 & 70 & 475 \\
\hline 33 & E41 & F2 & 240 & 0,449 & 299 & 2010 & 20 & 611 \\
\hline 34 & E42 & F2 & 420 & 0,539 & 235 & 2240 & 64,4 & 116 \\
\hline 35 & E43 & $\mathrm{F} 2$ & 240 & 0,042 & 217 & 848 & 23,1 & 879 \\
\hline 36 & E44 & F3 & 600 & 0,01 & 110 & 1130 & 17,3 & 1635 \\
\hline 37 & E45 & F3 & 780 & 0,044 & 289 & 1585 & 21,1 & 2100 \\
\hline 38 & E46 & Tr. Inf. & 280 & 0,053 & 18 & 95 & 27,6 & 368 \\
\hline 39 & E47 & Tr. Inf. & 300 & 0,181 & 56 & 213 & 14,7 & $>10000$ \\
\hline 40 & E48 & F3 & 1060 & 0,792 & 1335 & $>10000$ & 77,2 & $>10000$ \\
\hline 41 & E49 & F3 & 860 & $<0.005$ & 5 & 159 & 11,5 & 480 \\
\hline 42 & E50 & F3 & 1080 & 0,452 & 260 & 1505 & 10,6 & $>10000$ \\
\hline 43 & E51 & F3 & 1460 & 0,714 & 10 & 742 & 84,6 & 155 \\
\hline 44 & E52 & F3 & 800 & 0,639 & $>1500$ & $>10000$ & 53,5 & 1290 \\
\hline 45 & E53 & F3 & 1480 & 0,02 & 73 & 247 & 542 & 1730 \\
\hline 46 & E54 & F2 & 1100 & 2,03 & $>1500$ & 9080 & 23,1 & 5320 \\
\hline
\end{tabular}

Tableau 1 : Synthèse des résultats d'analyse de teneurs des principaux métaux sur les échantillons de minerai du Mourral de la Grave. Table 1: Synthesized results of principal metals ore grades analyses of the Mourral de la Grave samples. 
par des opérations minéralurgiques ${ }^{17}$, les mineurs pouvaient augmenter jusqu'à dix fois le taux de matière utile avant toute opération métallurgique. Parmi les teneurs des métaux convoités par les Anciens, les plus notables sont celles en $\mathrm{Cu}, \mathrm{Ag}$ et $\mathrm{Pb}$. Le cuivre est omniprésent et de manière générale sa teneur et sa distribution spatiale suivent la même logique que pour l'argent. Les teneurs les plus élevées en cuivre (parfois $>10000 \mathrm{~g} / \mathrm{t}$ ) ${ }^{18}$ correspondent aux endroits les plus riches en argent. Par ailleurs, les concentrations les plus élevées pour l'argent $(>1000 \mathrm{~g} / \mathrm{t})$ ont été détectées sur trois échantillons (E48, E52 et E54) de minerai massif pris dans la zone profonde du gisement, au croisement des filons F2 et F3. Des concentrations qui dépassent les $100 \mathrm{~g} / \mathrm{t}$ se rencontrent sur l'ensemble des travaux miniers étudiés, notamment aux croisements des filons, mais aussi dans les panneaux filoniens F2 et F3 et de manière plus localisée sur F1. Le plomb est aussi très répandu au sein du gisement du Mourral de la Grave, ses concentrations marquantes $(>10000 \mathrm{~g} / \mathrm{t})$ se positionnent aussi dans les zones profondes au croisement des filons F2 et F3, ainsi qu'aux croisements des filons au sein des Travaux inférieurs. La présence de l'or reste anecdotique, avec des teneurs négligeables, surtout pour les exploitations d'époques anciennes, entre 0,1 et $1 \mathrm{~g} / \mathrm{t}$ de minerai distribué sur l'ensemble du gisement. La plus importante concentration $(2,03 \mathrm{~g} / \mathrm{t})$ a été enregistrée sur l'échantillon E54. En ce qui concerne le fer, les valeurs obtenues sont trop médiocres $(<20 \%)$ pour pouvoir parler d'un véritable minerai de fer. Les plus fortes concentrations se placent dans la zone supérieure proche de la surface, la plus exposée aux phénomènes d'oxydation, lieu de formation des « chapeaux de fer ».

\section{Composition minéralogique des corps filoniens}

Les investigations géologiques des années 1970-1980 (Tollon, 1970; Crouzet et Tollon, 1980; Issard, 1984) classent le gisement du Mourral de la Grave de type épithermal à sulfures primaires $\left(S P C G^{19}\right)$, mis en place en deux étapes successives. Le premier épisode hydrothermal de moindre envergure a concerné une minéralisation à paragenèse arsénopyrite-pyrrhotite-bismuthinite-or. Plus active, la deuxième poussée hydrothermale, liée à un élargissement des failles, a mis en place des dépôts à dominante de carbonates, chalcopyrite, " cuivres gris ", galène et sphalérite. La paragenèse complète établie par F. Tollon est présentée dans le tableau 2.

17. Il s'agit d'opérations mécaniques (broyage-tri) et/ou thermomécaniques (grillage-broyage-tri) de traitement du minerai.

18. De manière à être plus parlantes les teneurs (massiques) des éléments métalliques ont été converties de ppm en g/t (idem dans les tableaux).

19. Sphalérite, pyrite, chalcopyrite, galène.

\begin{tabular}{|c|c|c|}
\hline $\begin{array}{l}\text { No } \\
\text { crt. }\end{array}$ & Minéral & Formule théorique \\
\hline 1 & arsénopyrite & FeAsS \\
\hline 2 & pyrrhotite & $\mathrm{Fe}_{7} \mathrm{~S}_{8}$ \\
\hline 3 & pyrite & $\mathrm{FeS}_{2}$ \\
\hline 4 & chalcopyrite & $\mathrm{CuFeS}_{2}$ \\
\hline 5 & sphalérite & $\mathrm{ZnS}$ \\
\hline 6 & galène & $\mathrm{PbS}$ \\
\hline 7 & bismuth natif & $\mathrm{Bi}$ \\
\hline 8 & bismuthinite & $\mathrm{Bi}_{2} \mathrm{~S}_{3}$ \\
\hline 9 & $\begin{array}{c}\text { cuivres gris (série tétraédrite- } \\
\text { tennantite) }\end{array}$ & $\begin{array}{l}\mathrm{Cu}_{6}\left[\mathrm{Cu}_{4}(\mathrm{Fe}, \mathrm{Zn})_{2}\right] \mathrm{Sb}_{4} \mathrm{~S}_{13}- \\
\mathrm{Cu}_{6}\left[\mathrm{Cu}_{4}(\mathrm{Fe}, \mathrm{Zn})_{2}\right] \mathrm{As}_{4} \mathrm{~S}_{13}\end{array}$ \\
\hline 10 & matildite & $\mathrm{AgBiS}_{2}$ \\
\hline 11 & pearcéite & $\begin{array}{c}\left(\left[\mathrm{Ag}_{9} \mathrm{CuS}_{4}\right]\right. \\
{\left[(\mathrm{Ag}, \mathrm{Cu})_{6}(\mathrm{As}, \mathrm{Sb})_{2} \mathrm{~S}_{7}\right]}\end{array}$ \\
\hline 12 & aïkinite & $\mathrm{CuPbBiS}_{3}$ \\
\hline 13 & semseyite & $\mathrm{Pb}_{9} \mathrm{Sb}_{8} \mathrm{~S}_{21}$ \\
\hline 14 & emplectite & $\mathrm{CuBiS}_{2}$ \\
\hline 15 & wittichenite & $\mathrm{Cu}_{3} \mathrm{BiS}_{3}$ \\
\hline 16 & rezbanyite & $\begin{array}{c}\text { non-valide IMA depuis } \\
1993 \\
\end{array}$ \\
\hline 17 & digénite & $\mathrm{Cu}_{1.8} \mathrm{~S}$ \\
\hline 18 & bornite & $\mathrm{Cu}_{5} \mathrm{FeS}_{4}$ \\
\hline 19 & covellite & $\mathrm{CuS}$ \\
\hline 20 & chalcocite & $\mathrm{Cu}_{2} \mathrm{~S}$ \\
\hline 21 & malachite & $\mathrm{Cu}_{2}\left(\mathrm{CO}_{3}\right)(\mathrm{OH})_{2}$ \\
\hline 22 & jalpaïte & $\mathrm{Ag}_{3} \mathrm{CuS}_{2}$ \\
\hline 23 & aragonite cuprifère & $\mathrm{CaCO}_{3}+\mathrm{Cu}$ \\
\hline
\end{tabular}

Tableau 2 : Paragenèse des minéraux métalliques du gisement des Barrencs proposé par F. Tollon (1970).

Table 2: Metal minerals paragenesis of the Barrencs ore deposit, proposed by F. Tollon (1970).

Les analyses que nous avons réalisées complètent la liste des variétés minérales des remplissages filoniens, mais surtout, elles apportent des éclaircissements sur leur qualité, leur fréquence et leur distribution spatiale sur l'ensemble de la zone étudiée, depuis l'affleurement en surface jusqu'aux profondeurs de la nappe phréatique (tableau 3).

La gangue filonienne est largement dominée par les minéraux carbonatés (dolomie, ankérite, calcite) et le quartz. Un phénomène généralisé de substitution ionique a été identifié par la présence significative de l'ankérite et de la sidérite dans la masse de l'encaissant carbonaté bordant les corps filoniens.

Dans la composition des reliquats de minerai des travaux anciens, à côté des minéraux mentionnés par les recherches antérieures, nos analyses microscopiques ont déterminé la 


\begin{tabular}{|c|c|c|c|c|c|}
\hline \multirow{2}{*}{$\mathrm{N}^{\circ} \mathrm{crt}$. } & \multirow{2}{*}{ Échantillon } & \multirow{2}{*}{ Microscopie } & \multirow{2}{*}{ Gangue } & \multicolumn{2}{|r|}{ Minéraux métalliques } \\
\hline & & & & $\%$ volume & Paragenèse \\
\hline 1 & E4 & $\begin{array}{l}\text { Optique } \\
\text { MEB } \\
\text { Microsonde }\end{array}$ & $\begin{array}{l}\text { dolomie, anké- } \\
\text { rite, quartz }\end{array}$ & 50 & $\begin{array}{l}\text { pyrite, chalcopyrite, galène }(+A g) \text {, sphalérite, freibergite, stromeyérite, } \\
\text { chalcocite }(+A g) \text {, ferrokestérite?, cérusite }\end{array}$ \\
\hline 2 & E13 & $\begin{array}{l}\text { Optique } \\
\text { MEB } \\
\text { Microsonde }\end{array}$ & dolomie, quartz & 23 & $\begin{array}{l}\text { freibergite, tétraédrite argentifère, chalcopyrite, covellite argentifère, yar- } \\
\text { rowite, stromeyérite, mackinstryite, acanthite, chlorargyrite }\end{array}$ \\
\hline 3 & E16 & $\begin{array}{l}\text { Optique } \\
\text { MEB } \\
\text { Microsonde }\end{array}$ & $\begin{array}{l}\text { ankérite, dolo- } \\
\text { mie, quartz }\end{array}$ & 32 & $\begin{array}{l}\text { pyrite, chalcopyrite }(+\mathrm{Ag}) \text {, galène }(+\mathrm{Ag}) \text {, freibergite, tennantite }(+\mathrm{Ag}) \text {, } \\
\text { stromeyérite, gustavite?, arcubisite?, vikingite?, cérusite, wustite, plomb } \\
\text { natif }\end{array}$ \\
\hline 4 & E17 & $\begin{array}{l}\text { Optique } \\
\text { MEB }\end{array}$ & $\begin{array}{l}\text { quartz, ankérite, } \\
\text { dolomie }\end{array}$ & 22 & Tétraédrite $(+\mathrm{Ag})$, chalcopyrite, pyrite, sphalérite, électrum, ténorite \\
\hline 5 & E18 & Optique & $\begin{array}{l}\text { quartz, ankérite, } \\
\text { dolomie }\end{array}$ & 40 & pyrite, chalcopyrite, " cuivre gris ", galène \\
\hline 6 & E26 & Optique & dolomie, quartz & 10 & pyrite, chalcopyrite, « cuivre gris ", galène \\
\hline 7 & E27 & Optique & $\begin{array}{l}\text { ankérite, dolo- } \\
\text { mie, quartz }\end{array}$ & 10 & pyrite, " cuivre gris ", chalcopyrite, galène, oxy-hydroxydes de fer \\
\hline 8 & E28 & Optique & $\begin{array}{l}\text { ankérite, dolo- } \\
\text { mie, quartz }\end{array}$ & 44 & pyrite, chalcopyrite, goethite \\
\hline 9 & E37 & $\begin{array}{l}\text { Optique } \\
\text { MEB } \\
\text { Microsonde }\end{array}$ & dolomie, quartz & 82 & $\begin{array}{l}\text { galène, galène }(+A g) \text {, chalcopyrite, chalcopyrite }(+A g) \text {, pyrite, sphalérite, } \\
\text { sphalérite }(+A g) \text {, tétraédrite }(+A g) \text {, bornite }(+A g) \text {, covellite }(+A g) \text {, reniérite? } \\
(+A g) \text {, furutobeite?, cérusite, oxy-hydroxydes de fer }\end{array}$ \\
\hline 10 & E38 & Optique & $\begin{array}{l}\text { dolomie, anké- } \\
\text { rite, quartz }\end{array}$ & - & pyrite, chalcopyrite, cuivre gris, sphalérite, galène, arsénopyrite \\
\hline 11 & E39 & Optique & $\begin{array}{l}\text { dolomie, anké- } \\
\text { rite, quartz }\end{array}$ & 22 & pyrite, chalcopyrite, galène, cuivre gris, sphalérite, arsénopyrite \\
\hline 12 & E40 & $\begin{array}{l}\text { Optique } \\
\text { MEB } \\
\text { Microsonde }\end{array}$ & $\begin{array}{l}\text { dolomie, anké- } \\
\text { rite, quartz }\end{array}$ & 22 & $\begin{array}{l}\text { pyrite }(+\mathrm{Ag}) \text {, arsénopyrite, galène }(+\mathrm{Ag}) \text {, pearcéite, « cuivre gris ", ferroskut- } \\
\text { terudite?, goethite }\end{array}$ \\
\hline 13 & E41 & $\begin{array}{l}\text { Optique } \\
\text { MEB }\end{array}$ & $\begin{array}{l}\text { dolomie, anké- } \\
\text { rite, quartz }\end{array}$ & 44 & $\begin{array}{l}\text { pyrite, chalcopyrite, tétraédrite }(+\mathrm{Ag}) \text {, arsénopyrite?, galène, digénite }(+\mathrm{Ag}) \text {, } \\
\text { covellite }(+\mathrm{Ag}) \text {, chalcocite }(+\mathrm{Ag}) \text {, bornite }(+\mathrm{Ag}) \text {, azurite }\end{array}$ \\
\hline 14 & E47 & Optique & quartz & 60 & galène, pyrite, sphalérite, cérusite \\
\hline 15 & E48 & $\begin{array}{l}\text { Optique } \\
\text { MEB } \\
\text { Microsonde }\end{array}$ & quartz, dolomie & 88 & $\begin{array}{l}\text { sphalérite, pyrite, chalcopyrite, galène }(+\mathrm{Ag}) \text {, freibergite, acanthite, quadra- } \\
\text { tite?, cérusite }\end{array}$ \\
\hline 16 & E50 & $\begin{array}{l}\text { Optique } \\
\text { Microsonde }\end{array}$ & quartz & 15 & galène $(+\mathrm{Ag})$, freibergite, chalcopyrite, pyrite, électrum \\
\hline 17 & E51 & $\begin{array}{l}\text { Optique } \\
\text { MEB }\end{array}$ & quartz, ankérite & 70 & pyrite, chalcopyrite $(+A g)$, sphalérite $(+A g)$, galène \\
\hline 18 & E53 & Optique & ankérite & 10 & pyrite, galène, " cuivres gris ", chalcopyrite, oxy-hydroxydes de fer \\
\hline 19 & E54 & $\begin{array}{l}\text { Optique } \\
\text { Microsonde }\end{array}$ & quartz & 89 & $\begin{array}{l}\text { freibergite, tétraédrite }(+\mathrm{Ag}) \text {, bournonite }(+\mathrm{Ag}) \text {, chalcopyrite, sphalérite, } \\
\text { pyrite, cérusite }(+\mathrm{Ag})\end{array}$ \\
\hline
\end{tabular}

Tableau 3 : Échantillons analysés en microscopie - composition de la gangue - participation volumique et paragenèse des minéraux métalliques.

Table 3: Microscope samples analyses - Gangue composition - volume participation and metallic minerals paragenesis.

présence d'autres sulfures de cuivre-argent ${ }^{20}$ et de sulfosel ${ }^{21}$. Cela a aussi permis de constater la présence de minéraux complexes de cuivre, argent, plomb, fer et zinc (minéraux avec points d'interrogation dans tableaux 4 et 5), pour les-

20. Yarrowite, stromeyérite, mackinstryite.

21. Bournonite, gustavite, vikingite. quels des analyses supplémentaires (notamment cristallographiques) devront être réalisées pour certifier leur existence.

Les résultats des analyses (tableaux 4 et 5) montrent que l'argent forme des sulfures et des sulfosels complexes avec le cuivre et le plomb ${ }^{22}$. Dans la famille des « cuivres gris ", très

22. Pearcéite, stromeyérite, mackinstryite, gustavite, vikingite. 


\begin{tabular}{|c|c|c|c|c|c|c|c|}
\hline \multicolumn{2}{|l|}{ Minéral métallique } & \multirow{2}{*}{ Échant. } & \multirow{2}{*}{$\mathrm{N}^{\circ}$ analyses } & \multicolumn{4}{|c|}{ Éléments (pourcentage/poids normalisé) W\% min-max } \\
\hline Nom & Formule chimique théorique & & & $\mathrm{Cu}$ & $\mathrm{Ag}$ & $\mathrm{Fe}$ & $\mathrm{Pb}$ \\
\hline \multirow{7}{*}{ Freibergite } & \multirow{7}{*}{$\mathrm{Ag}_{6}\left[\mathrm{Cu}_{4} \mathrm{Fe}_{2}\right] \mathrm{Sb}_{4} \mathrm{~S}_{13-x}$} & E4 & 3 & $31,58-47,61$ & $7,15-13,34$ & $2,01-3,99$ & 0 \\
\hline & & \multirow{2}{*}{ E13 } & 1 & 32,23 & 32,19 & 1,75 & 5,13 \\
\hline & & & 2 & $36,12-36,70$ & $2,20-2,58$ & & 0 \\
\hline & & E16 & 2 & $17,15-28,98$ & $12,22-26,35$ & $4,61-6,98$ & 0 \\
\hline & & E17 & 4 & $35,16-36,42$ & $3,12-5,16$ & $4,28-4,69$ & 0 \\
\hline & & E37 & 3 & $36,03-46,88$ & $1,92-2,83$ & $2,30-6,21$ & 6,21 \\
\hline & & E48 & 6 & $24,05-33,39$ & $9,02-21,26$ & $3,73-4,11$ & 0 \\
\hline Tétraédrite argentifère & $\mathrm{Cu}_{6}\left[\mathrm{Cu}_{4}(\mathrm{Fe}, \mathrm{Zn})_{2}\right] \mathrm{Sb}_{4} \mathrm{~S}_{13}$ & E41 & 1 & 39,33 & 0,29 & 1,88 & 0 \\
\hline Tennantite argentifère & $\mathrm{Cu}_{6}\left[\mathrm{Cu}_{4}(\mathrm{Fe}, \mathrm{Zn})_{2}\right] \mathrm{As}_{4} \mathrm{~S}_{13}$ & E16 & 1 & 19,42 & 2,17 & 25,64 & 0 \\
\hline \multirow{3}{*}{ Chalcopyrite } & \multirow{3}{*}{$\mathrm{CuFeS}_{2}$} & E16 & 2 & $30,84-35,03$ & 0 & $29,91-30,66$ & 0 \\
\hline & & E37 & 2 & $33,71-33,93$ & $0-0,65$ & $31,05-31,44$ & 0 \\
\hline & & E51 & 1 & 32,38 & 0,28 & 26,78 & 0 \\
\hline Pyrite & $\mathrm{FeS}_{2}$ & E16 & 1 & 0 & 0 & 43,41 & 0 \\
\hline Galène argentifère & $\mathrm{PbS}$ & E48 & 1 & 0,6 & 13,24 & 0 & 73,82 \\
\hline \multirow{2}{*}{ Galène } & \multirow{2}{*}{$\mathrm{PbS}$} & E16 & 1 & 1,91 & 0 & 1,52 & 84,04 \\
\hline & & E51 & 1 & 0 & 0 & 8,64 & 76,81 \\
\hline \multirow{3}{*}{ Sphalérite } & \multirow{3}{*}{$\mathrm{ZnS}$} & E37 & 2 & $0-8,09$ & $0-0,11$ & $1,33-7,77$ & 0 \\
\hline & & E48 & 2 & $0-1,82$ & 0 & $1,95-2,62$ & 0 \\
\hline & & E51 & 1 & 8,72 & 0,79 & 7,55 & 0 \\
\hline Arsénopyrite & FeAsS & E40 & 1 & 0 & 0 & 36,82 & 0 \\
\hline \multirow{3}{*}{ Covellite argentifere } & \multirow{3}{*}{$\mathrm{CuS}$} & E13 & 1 & 61,7 & 9,84 & 0 & 0 \\
\hline & & E37 & 1 & 61,98 & 4,22 & 1 & 0 \\
\hline & & E41 & 1 & 65,45 & 4,75 & 0,59 & 0 \\
\hline \multirow{2}{*}{ Chalcocite argentifère } & \multirow{2}{*}{$\mathrm{Cu}_{2} \mathrm{~S}$} & E4 & 1 & 70,21 & 4,28 & 2,05 & 0 \\
\hline & & E41 & 2 & $73,41-76,34$ & $0,29-1,12$ & $1,17-3,85$ & 0 \\
\hline Digénite argentifere & $\mathrm{Cu}_{1.8} \mathrm{~S}$ & E41 & 5 & $68,17-76,51$ & $0,34-1,78$ & $0,40-2,08$ & 0 \\
\hline Yarrowite & $\mathrm{Cu}_{9} \mathrm{~S}_{8}$ & E13 & 1 & 69,21 & 0 & 0,55 & 0 \\
\hline \multirow{3}{*}{ Stromeyérite } & \multirow{3}{*}{$\mathrm{CuAgS}$} & E4 & 4 & $24,01-46,83$ & $28,77-44,77$ & $1,77-6,78$ & 0 \\
\hline & & E13 & 1 & 50,71 & 18,03 & 1,71 & 0 \\
\hline & & E16 & 1 & 26,15 & 52,82 & 0 & 0 \\
\hline Mackinstryite & $(\mathrm{Ag}, \mathrm{Cu})_{2} \mathrm{~S}$ & E13 & 1 & 23,48 & 61,93 & 5,79 & 0 \\
\hline Pearcéite & {$\left[\mathrm{Ag}_{9} \mathrm{CuS}_{4}\right]\left[(\mathrm{Ag}, \mathrm{Cu})_{6}(\mathrm{As}, \mathrm{Sb})_{2} \mathrm{~S}_{7}\right]$} & E40 & 1 & 4,65 & 60,85 & 6,11 & 0 \\
\hline Bornite argentifère & $\mathrm{Cu}_{5} \mathrm{FeS}_{4}$ & E41 & 1 & 61,48 & 2,1 & 6,02 & 0 \\
\hline Gustavite & $\mathrm{AgPbBi}_{3} \mathrm{~S}_{6}$ & E16 & 1 & 0 & 8,61 & 0 & 19,65 \\
\hline Vikingite & $\mathrm{Ag}_{5} \mathrm{~Pb}_{8} \mathrm{Bi}_{13} \mathrm{~S}_{30}$ & E16 & 1 & 1,96 & 9,53 & 1,18 & 25,49 \\
\hline électrum & $\mathrm{Au}_{1,5} \mathrm{Ag}$ & E17 & 2 & $1,30-2,24$ & $21,11-22,39$ & 0 & 0 \\
\hline furutobéite? & $(\mathrm{Cu}, \mathrm{Ag})_{6} \mathrm{PbS}_{4}$ & E37 & 1 & 40,95 & 2,51 & 1,04 & 30,53 \\
\hline arcubisite? & $\mathrm{Ag}_{6} \mathrm{CuBiS}_{4}$ & E16 & 1 & 0,83 & 57,94 & 0 & 0 \\
\hline ferrokestérite? & $\mathrm{Cu}_{2}(\mathrm{Fe}, \mathrm{Zn}) \mathrm{SnS}_{4}$ & E4 & 1 & 16,73 & 0 & 9,04 & 0 \\
\hline ferroskutterudite? & $(\mathrm{Fe}, \mathrm{Co}) \mathrm{As}_{3}$ & E40 & 1 & 0 & 0 & 12,14 & 0 \\
\hline
\end{tabular}

Tableau 4 : Minéraux détectés à l'issue des analyses au MEB et participation quantitative min-max (pourcentage/poids normalisé-W\%) des principaux métaux (les sulfures communes majoritaires, PSCG, ont fait rarement l'objet d'analyses quantitatives).

Table 4: Detected minerals (common sulphides, PSCG, were not systematically analysed) with SEM analyses with min-max main metals' quantitative participation (normalised weight percentage-W\%). 


\begin{tabular}{|c|c|c|c|c|c|c|c|}
\hline \multirow{2}{*}{ Minéral } & \multirow{2}{*}{ Éch. } & \multirow{2}{*}{$\mathrm{N}^{\circ}$ analyses } & \multicolumn{4}{|c|}{ Éléments (pourcentage/poids - W\% min-max) } & \multirow{2}{*}{ Composition chimique moyenne calculée } \\
\hline & & & $\mathrm{Cu}$ & $\mathrm{Ag}$ & $\mathrm{Fe}$ & $\mathrm{Pb}$ & \\
\hline \multirow{6}{*}{ Freibergite } & \multirow{2}{*}{$\mathrm{E} 4$} & 7 & $32,35-34,73$ & $4,29-7,45$ & $2,16-4,12$ & 0 & $\left(\mathrm{Cu}_{8,96} \mathrm{Fe}_{0,91} \mathrm{Zn}_{1,23} \mathrm{Ag}_{1,04}\right)_{12,14}\left(\mathrm{Sb}_{3,62} \mathrm{As}_{0,13} \mathrm{Bi}_{0,02}\right)_{3,77} \mathrm{~S}_{13,09}$ \\
\hline & & 4 & $30,05-31,77$ & $7,61-9,01$ & $3,07-3,31$ & 0 & $\left(\mathrm{Cu}_{8,54} \mathrm{Fe}_{1,01} \mathrm{Zn}_{1,19} \mathrm{Ag}_{1,35}\right)_{12,09}\left(\mathrm{Sb}_{3,62} \mathrm{As}_{0,08} \mathrm{Bi}_{0,01}\right)_{4,01} \mathrm{~S}_{13,22}$ \\
\hline & E16 & 2 & $25,21-26,56$ & $13,41-17,32$ & $4,83-5,71$ & 0 & $\left(\mathrm{Cu}_{7,18} \mathrm{Fe}_{1,66} \mathrm{Zn}_{0,64} \mathrm{Ag}_{2,52}\right)_{12}\left(\mathrm{Sb}_{3,78} \mathrm{As}_{0,1} \mathrm{Bi}_{0,05}\right)_{3,93} \mathrm{~S}_{12,87}$ \\
\hline & E48 & 11 & $22,75-28,55$ & $11,49-20,64$ & $3,20-4,85$ & 0 & $\left.\mathrm{Cu}_{6,79} \mathrm{Fe}_{1,16} \mathrm{Zn}_{0,9} \mathrm{Ag}_{3,09}\right)_{11,94}\left(\mathrm{Sb}_{3,77} \mathrm{As}_{0,21}\right)_{3,98} \mathrm{~S}_{13,08}$ \\
\hline & E50 & 23 & $26,71-28,58$ & $12,96-15,25$ & $3,09-5,56$ & 0 & $\left(\mathrm{Cu}_{7,64} \mathrm{Fe}_{1,12} \mathrm{Zn}_{0,85} \mathrm{Ag}_{2,26}\right)_{11,87}\left(\mathrm{Sb}_{3,84} \mathrm{As}_{0,12}\right)_{3,96} \mathrm{~S}_{13,14}$ \\
\hline & E54 & 10 & $32,82-36,59$ & $1,70-7,09$ & $3,80-4,48$ & 0 & $\left(\mathrm{Cu}_{9,16} \mathrm{Fe}_{1,25} \mathrm{Zn}_{0,64} \mathrm{Ag}_{0,86}\right)_{11,91}\left(\mathrm{Sb}_{3,77} \mathrm{As}_{0,13}\right)_{3,9} \mathrm{~S}_{13,14}$ \\
\hline $\begin{array}{l}\text { Tétraédrite } \\
\text { argentiferre }\end{array}$ & E37 & 8 & $36,41-37,79$ & $0,89-1,66$ & $1,84-2,86$ & 0 & $\left(\mathrm{Cu}_{9,7}, \mathrm{Fe}_{0,64} \mathrm{Zn}_{1,25} \mathrm{Ag}_{0,19}\right)_{11,78}\left(\mathrm{Sb}_{3,92} \mathrm{As}_{0,13}\right)_{4,05} \mathrm{~S}_{13,16}$ \\
\hline \multirow{5}{*}{ Chalcopyrite } & E4 & 1 & 33,54 & 0,04 & 31,38 & 0 & $\mathrm{Cu}_{0,96} \mathrm{Fe}_{1,02} \mathrm{~S}_{2,02}$ \\
\hline & E16 & 6 & $32,60-34,88$ & $0,07-0,12$ & $28,78-30,70$ & 0 & $\mathrm{Cu}_{0,99} \mathrm{Fe}_{0,99} \mathrm{~S}_{2,01}$ \\
\hline & E37 & 7 & $33,57-34,13$ & $0,09-0,61$ & $28,03-29,75$ & 0 & $\left(\mathrm{Cu}_{0,99} \mathrm{Ag}_{0,01} \mathrm{Zn}_{0,01}\right)_{1,01} \mathrm{Fe}_{0,97} \mathrm{~S}_{2,03}$ \\
\hline & E50 & 8 & $32,56-34,36$ & $0-0,18$ & $27,03-29,97$ & 0 & $\mathrm{Cu}_{0,99} \mathrm{Fe}_{0,97} \mathrm{~S}_{2,04}$ \\
\hline & $\mathrm{E} 54$ & 4 & $34,25-34,40$ & $0,08-0,29$ & $29,62-30,00$ & 0 & $\left(\mathrm{Cu}_{0,99} \mathrm{Ag}_{0,01}\right) \mathrm{Fe}_{0,98} \mathrm{~S}_{2,02}$ \\
\hline \multirow{7}{*}{ Pyrite } & E4 & 2 & $0-0,68$ & 0 & $46,37-47,91$ & 0 & $\left(\mathrm{Fe}_{1,01} \mathrm{Cu}_{0,01} \mathrm{Zn}_{0,01} \mathrm{Bi}_{0,01}\right)_{1,03} \mathrm{~S}_{1,97}$ \\
\hline & E16 & 5 & $0-0,77$ & $0-0,04$ & $47,15-47,90$ & 0 & $\left(\mathrm{Fe}_{1,01} \mathrm{As}_{0,01} \mathrm{Bi}_{0,01}\right)_{1,03} \mathrm{~S}_{1,97}$ \\
\hline & E37 & 2 & $0,01-0,22$ & 0 & $45,77-46,23$ & 0 & $\mathrm{Fe}_{0,99} \mathrm{~S}_{2}$ \\
\hline & $\mathrm{E} 40$ & 3 & $0,01-0,12$ & $0,04-0,64$ & $44,30-46,22$ & 0 & $\mathrm{Fe}_{0,98}\left(\mathrm{Ag}, \mathrm{As}, \mathrm{Bi}_{0,02} \mathrm{~S}_{2}\right.$ \\
\hline & $\mathrm{E} 48$ & 2 & $0-0,02$ & $0,01-0,06$ & $46,12-46,30$ & 0 & $\mathrm{FeS}_{2}$ \\
\hline & E50 & 3 & $0-0,27$ & $0-0,07$ & $43,60-44,73$ & 0 & $\left(\mathrm{Fe}_{0,96} \mathrm{Cu}_{0,01} \mathrm{Bi}_{0,01}\right)_{0,98} \mathrm{~S}_{2,02}$ \\
\hline & E54 & 1 & 0,36 & 0 & 45,56 & 0 & $\left(\mathrm{Fe}_{0,99} \mathrm{Cu}_{0,01}\right) \mathrm{S}_{2}$ \\
\hline \multirow{5}{*}{$\begin{array}{l}\text { Galène argen- } \\
\text { tifere }\end{array}$} & E 4 & 4 & $0-0,64$ & $0,58-0,93$ & $0,12-1,18$ & $82,47-84,71$ & $\left(\mathrm{~Pb}_{0,93} \mathrm{Fe}_{0,3} \mathrm{Ag}_{0,02} \mathrm{Bi}_{0,02}\right) \mathrm{S}$ \\
\hline & E16 & 1 & 0 & 1,21 & 0 & 80,27 & $\left(\mathrm{~Pb}_{0,92} \mathrm{Ag}_{0,03} \mathrm{Bi}_{0,03}\right)_{0,98} \mathrm{~S}_{1,02}$ \\
\hline & E37 & 8 & $0-0,16$ & $0-0,2$ & 0 & $88,66-89,28$ & $\mathrm{~Pb}_{0,98} \mathrm{~S}$ \\
\hline & E48 & 15 & $0-0,17$ & $0-0,14$ & 0 & $86,44-88,83$ & $\mathrm{~Pb}_{0,99} \mathrm{~S}$ \\
\hline & E50 & 14 & $0-0,71$ & $0,44-1,96$ & 0 & $80,65-86,42$ & $\left(\mathrm{~Pb}_{0,95} \mathrm{Ag}_{0,02} \mathrm{Cu}_{0,01}\right)_{0,98} \mathrm{~S}_{1,02}$ \\
\hline \multirow{3}{*}{ Sphalérite } & E4 & 2 & $0,11-028$ & $0-0,05$ & $1,05-1,24$ & 0 & $\left(\mathrm{Zn}_{0,95} \mathrm{Fe}_{0,02} \mathrm{Cu}_{0,01} \mathrm{Bi}_{0,01}\right)_{0,99} \mathrm{~S}_{1,01}$ \\
\hline & $\mathrm{E} 37$ & 9 & $0,02-5,22$ & $0-0,51$ & $0,49-7,79$ & $0-0,73$ & $\left(\mathrm{Zn}_{0,88} \mathrm{Fe}_{0,05} \mathrm{Cu}_{0,04} \mathrm{Cd}_{0,01} \mathrm{~Pb}_{0,01}\right)_{0,99} \mathrm{~S}$ \\
\hline & E48 & 2 & $2,45-3,47$ & $0-0,03$ & $3,70-3,87$ & 0 & $\left(\mathrm{Zn}_{0,86} \mathrm{Fe}_{0,07} \mathrm{Cu}_{0,05}\right)_{0,98} \mathrm{~S}$ \\
\hline Arsénopyrite & $\mathrm{E} 40$ & 2 & 0,01 & $0,01-0,04$ & $36,35-36,64$ & 0 & $\mathrm{Fe}_{0,94} \mathrm{As}_{0,73} \mathrm{~S}_{1,32}$ \\
\hline $\begin{array}{c}\text { Covellite } \\
\text { argentifere }\end{array}$ & $\mathrm{E} 37$ & 4 & $42,00-53,05$ & $0,90-3,54$ & $3,72-10,86$ & $1,112,51$ & $\left(\mathrm{Cu}_{0,84} \mathrm{Fe}_{0,15} \mathrm{Ag}_{0,03} \mathrm{~Pb}_{0,02} \mathrm{Zn}_{0,01} \mathrm{Sb}_{0,01}\right)_{1,05} \mathrm{~S}$ \\
\hline Stromeyérite & E4 & 1 & 27,74 & 42,29 & 5,65 & 4,97 & $\left(\mathrm{Cu}_{0,83} \mathrm{Fe}_{0,19} \mathrm{~Pb}_{0,05} \mathrm{Ni}_{0,01} \mathrm{As}_{0,01}\right)_{1,09} \mathrm{Ag}_{0,75} \mathrm{~S}_{1,16}$ \\
\hline Bournotite & E54 & 5 & $12,37-12,80$ & $0-0,2$ & & $40,67-42,24$ & $\mathrm{~Pb}_{0,98}\left(\mathrm{Cu}_{0,95} \mathrm{Ag}_{0,01}\right)_{0,96}\left(\mathrm{Sb}_{0,98} \mathrm{As}_{0,01}\right)_{0,99} \mathrm{~S}_{3,07}$ \\
\hline Gustavite & E16 & 3 & $0,05-0,06$ & $9,32-9,65$ & $0-0,08$ & $18,57-19,24$ & $\left(\mathrm{Fe}_{0,01} \mathrm{~Pb}_{1,01}\right)_{1,02}\left(\mathrm{Ag}_{0,96} \mathrm{Cu}_{0,01}\right)_{0,97}\left(\mathrm{Bi}_{2,62} \mathrm{Sb}_{0,24}\right)_{2,86} \mathrm{~S}_{6,15}(?)$ \\
\hline Lillianite? & E16 & 2 & $0,04-0,08$ & $7,00-7,20$ & 0 & $39,34-41,47$ & $\mathrm{~Pb}_{2,32}\left(\mathrm{Ag}_{0,79} \mathrm{Cu}_{0,01}\right)_{0,8}\left(\mathrm{Bi}_{1,81} \mathrm{As}_{0,1} \mathrm{Sb}_{0,13}\right)_{1,95} \mathrm{~S}_{5,93}(?)$ \\
\hline Lillianite? & E16 & 1 & 2,04 & 2,54 & 2,12 & 54,94 & $\left(\mathrm{Fe}_{0,55} \mathrm{~Pb}_{3,85}\right)_{4,4}\left(\mathrm{Ag}_{0,34} \mathrm{Cu}_{0,46}\right)_{0,8} \mathrm{Bi}_{0,61} \mathrm{~S}_{5,18}$ (?) \\
\hline
\end{tabular}

Tableau 5 : Synthèse des analyses à la microsonde - minéraux détectés - participation quantitative min-max (poucentage/poids - W\%) des principaux métaux et composition chimique moyenne calculée.

Table 5: EPMA analyses results - detected minerals - min-max main metals' quantitative participation (weight percentage-W\%) and calculated average chemical composition.

répandus au sein du gisement, nous constatons une prédominance des variétés riches en argent : la freibergite et la tétraédrite argentifère et, en moindre mesure, la tennantite argentifère. Mais, à une plus faible teneur, l'argent est inclus dans d'autres sulfures, notamment dans la galène, dans les sulfures de cuivre ${ }^{23}$ et dans la bournonite. Parmi les sulfures

23. Chalcopyrite, covellite, bornite, chalcocite, digénite. porteurs du cuivre, la chalcopyrite est dominante mais le cuivre est contenu dans une large variété de sulfures ${ }^{24}$ et de sulfosel $\mathrm{s}^{25}$. Le principal minéral porteur du plomb est la galène et il se retrouve secondairement dans d'autres miné-

24. Covellite, chalcocite, digénite, yarrowite, bornite, stromeyérite, mackinstrite.

25. "Cuivres gris », bournonite, pearcéite. 
raux, notamment la bournonite, ainsi que dans les sulfosels à bismuth ${ }^{26}$. Les principaux minéraux primaires de fer sont la pyrite, la chalcopyrite et l'arsénopyrite, mais dans une mesure plus ou moins importante, le fer participe dans la composition de la plupart des minéraux détectés.

Les phénomènes d'altération exogène (oxydation) se sont propagés, tout d'abord, dans les parties du gisement situées à l'interface avec l'atmosphère et, ultérieurement, en profondeur, à travers les vides créés par l'exploitation minière. Ainsi une importante partie des reliquats de minerai sur les parements et les piliers des ouvrages miniers ont été exposés à l'oxydation. Les observations de terrain corroborées par les analyses en laboratoire, montrent une participation importante des produits secondaires, carbonates-sulfates-oxydeshydroxydes ${ }^{27}$, issus de l'oxydation supergène des produits polymétalliques primaires et de la karstification post-exploitation (tableau 3).

Dans l'ensemble, la composition minéralogique des minerais du gisement du Mourral de la Grave est dominée par la pyrite, la chalcopyrite, les « cuivres gris » argentifères, la galène et la galène argentifere, la sphalérite, des sulfures de cuivre souvent argentifères et, secondairement, des sulfures et sulfosels complexes d'argent, cuivre et plomb. Il ressort que pour les anciens mineurs, la minéralisation dominante du gisement est le cuivre, avec une participation importante de l'argent et du plomb.

\section{L'influence des facteurs gîtologiques et minéralogiques sur l'architecture et la dynamique de l'exploitation}

De nos jours, les vastes espaces ouverts par les travaux miniers souterrains de toutes époques offrent une lecture aisée du gisement et facilitent l'étude gîtologique du site des Barrencs et, notamment, du groupe filonien du Mourral de la Grave. À l'époque de l'ouverture des filons, les anciens mineurs ne bénéficiaient pas d'une vue d'ensemble, à l'exception de la géométrie visible à l'affleurement des filons. De ce fait, l'organisation et la stratégie d'exploitation étaient déterminées par l'avancement des travaux en profondeur et s'adaptèrent à la forme et à la richesse des minéralisations, ainsi qu'aux contraintes techniques et de gestion de l'exploitation.

26. Gustavite, vikingite, etc.

27. Produits secondaires : ankérite $\left(\mathrm{Ca}\left(\mathrm{Fe}^{2+}, \mathrm{Mg}\right)\left(\mathrm{CO}_{3}\right)_{2}\right)$, sidérite $\left(\mathrm{Fe}\left(\mathrm{CO}_{3}\right)\right)$, malachite $\left(\mathrm{Cu}_{2}\left(\mathrm{CO}_{3}\right)(\mathrm{OH})_{2}\right)$, azurite $\left(\mathrm{Cu}_{3}\left(\mathrm{CO}_{3}\right) 2(\mathrm{OH})_{2}\right)$, cérusite $\left(\mathrm{Pb}\left(\mathrm{CO}_{3}\right)\right)$, anglésite $(\mathrm{Pb}(\mathrm{SO} 4))$, chalcanthite? $\left(\mathrm{Cu}\left(\mathrm{SO}_{4}\right) 5 \mathrm{H}_{2} \mathrm{O}\right)$, goethite $(\mathrm{FeO}(\mathrm{OH}))$, wustite $(\mathrm{FeO})$, cuprite $\left(\mathrm{Cu}_{2} \mathrm{O}\right)$, ténorite $(\mathrm{CuO})$, arsénolite $\left(\mathrm{As}_{2} \mathrm{O}_{3}\right)$.
Le démarrage de l'exploitation nécessitait évidement une prospection des affleurements en pratiquant des sondages pour tester la richesse du gisement. La plupart des indices de prospection ou les ouvrages à la surface de la structure filonienne du Mourral de la Grave sont totalement comblés par les éboulis de pente et recouverts par la végétation. Tout de même, l'étude du réseau souterrain menée conjointement à la prospection en surface nous a permis d'identifier six orifices et/ou chantiers connectés aux travaux souterrains étudiés, mais aujourd'hui obstrués (figure 2a). De nombreuses autres recherches de ce type doivent se trouver le long de l'affleurement filonien sur le versant sud, ainsi que dans l'allongement nord, sur le plateau.

Au fur et à mesure de l'approfondissement des ouvrages à l'intérieur du gisement, la stratégie de recherche des zones minéralisées employée par les Anciens reste dans les limites latérales de la structure filonienne du Mourral de la Grave. Le relevé topographique (figure 2a) dessine une trame de galeries de prospection quasi régulièrement étagées et reliées par des puits, développée de façon unidirectionnelle dans les plans filoniens. Les reliquats d'anciens niveaux visibles dans les chantiers d'exploitation et les galeries de recherche dans les zones qui n'ont pas abouti à une exploitation (en général stériles ou pauvres), montrent un étagement tous les 3 à $5 \mathrm{~m}$. Parfois les recherches ont porté sur des branches filoniennes secondaires plus ou moins minéralisées et même sur des failles et fissures à remplissage stérile où les galeries ont été foncées sur 10 , voire $15 \mathrm{~m}$ de longueur (figures $3 \mathrm{c}$ et $5 \mathrm{c}$ ).

Dans le plan latéral, la limite Ouest des travaux miniers est matérialisée en général par le filon F1, les travaux les plus profonds reconnaissables sur ce filon s'arrêtent au contact avec le filon F2. Le flanc oriental est limité généralement par les ouvrages d'exploitation du filon F3. Mais le plan du filon F2 et, par endroits, les travaux miniers conséquents dépassent latéralement de peu les plans des panneaux filoniens (figures $3 \mathrm{~b}$ et $\mathrm{c}$ ). Le filon transversal $\mathrm{F} 4$ est de moindre importance car confiné par les trois autres, il est exploité au contact avec les filons F2 et F3 et les travaux s'avancent sur quelques mètres côté mur du filon F2 (figure $3 \mathrm{~b}$ - coupe 2). La part importante des calcschistes dans la partie méridionale des Travaux supérieurs a induit une minéralisation plus vaste. Elle dépasse le cadre des panneaux filoniens principaux et pénètre en profondeur l'encaissant le long des plans de schistosité, failles et fissures secondaires. La cohésion faible de l'encaissant schisteux, l'omniprésence de failles et de fissures secondaires, ainsi que l'extension des travaux miniers à la recherche du minerai dans la masse de l'encaissant ont entrainé une déstabilisation dans l'ensemble des ouvrages. Cela a inévitablement provoqué l'effondrement 
perçu actuellement dans la plus grande partie de ce secteur (figure 3a - côté sud du Grand chantier).

La recherche de la minéralisation filonienne a été réalisée, à maints endroits, par un creusement qui passe d'un côté à l'autre de la faille ou fissure directrice du filon, afin de reconnaitre la diffusion de la minéralisation dans l'encaissant (figure 5d). De son côté, l'étude archéométrique réalisée sur les gabarits et les formes des sections des galeries de recherche ou d'assistance a apporté d'autres indices qui montrent à quel point cette prospection avait été menée d'une manière organisée, systématique et efficace. Quelle que soit la technique d'abattage employée (attaque par le feu, à l'outil ou mixte), le gabarit des galeries était standardisé, en moyenne de $1 \mathrm{~m}$ de hauteur sur $0,80 \mathrm{~m}$ de largeur et de forme ovoïde à rectangulaire aux angles arrondis. Du point de vue géologique, dans tous les points mesurés, les galeries suivaient des filons ou des accidents géologiques (faille, fissure) et, dans plusieurs cas, le mineur s'aidait du miroir de faille pour faciliter l'avancement, donnant ainsi une forme "découpée » à la section de la galerie (figure $2 \mathrm{~d}$ ).

Une fois que les travaux étaient avancés sur des panneaux ou branches filoniennes parallèles, à plusieurs endroits, les mineurs les ont reliés par des galeries d'assistance en traversbanc. Un exemple qui montre la grande compétence des mineurs anciens et leur connaissance empirique de la géologie est celui de la jonction en travers-banc entre le Chantier supérieur nord (sur le filon F2) et le secteur étudié au nord du Grand Chantier (sur le filon F3). Conscients que les travaux menés séparément dans les deux secteurs avaient avancé à un stade de proximité et désirant les rejoindre, les mineurs ont tenté la jonction à quatre endroits (figure $2 \mathrm{a}: \mathrm{Z} 1$ et $3 \mathrm{~b}$ : coupe 2). Deux des tentatives, une d'entre elles réussie, ont été réalisées sur la faille directrice du filon F4. Ainsi les mineurs étaient très probablement conscients que ce filon, exploité dans sa partie supérieure au croisement avec le F2, butait dans le filon F3.

Une fois la reconnaissance du gisement réalisée par le biais des galeries de recherche, les zones riches étaient ensuite exploitées du haut vers le bas par la méthode des gradins droits, et les massifs minéralisés compris entre deux niveaux de recherche étaient alors enlevés. Mais l'abattage des zones riches ne s'effectuait pas au hasard. L'action était coordonnée de manière à ce que l'exploitation d'une zone ne puisse pas nuire à l'avancement sur l'ensemble de la mine. Cette démarche est bien lisible en haut du Chantier supérieur sud (figure 2a : Z2).

Les analyses des échantillons de minerai montrent systématiquement que les élargissements des ouvrages correspondent à un enrichissement du minerai en $\mathrm{Cu}$ et $\mathrm{Ag}$, voire en $\mathrm{Pb}$, et à un accroissement de la puissance de la minérali- sation, la forme de l'abattage (chantier) épousant la géométrie du corps filonien. De temps en temps, les mineurs ont laissé en place des massifs minéralisés (piliers) pour assurer le soutènement des épontes des chantiers. Même les enrichissements peu étendus, à quelques exceptions près, ont donné lieu à des petits chantiers. Un exemple éloquent est fourni par les deux petits chantiers situés sur le plus haut niveau de recherche étudié (+318) (figure $2 \mathrm{a}: \mathrm{Z3}$ ), sur le filon F1, filon très peu exploité par ailleurs.

Dans leur ensemble, les travaux miniers anciens du Mourral de la Grave ont exploité des panneaux filoniens, successifs et redressés, depuis l'affleurement en surface vers les profondeurs (figure $2 \mathrm{c}$ ). La totalité des travaux anciens se développe dans l'allongement des structures filoniennes. Sur l'ensemble des travaux étudiés, aucun indice d'ouvrage en travers-banc n'a été observé sur les flancs extérieurs. Le seul connu est celui de Lastours (116 m de longueur) appartenant aux recherches du $\mathrm{xx}^{\mathrm{e}}$ siècle. Cette stratégie d'exploitation peut être expliquée d'abord par la position du gisement par rapport à la configuration locale du relief (figures 1a et c). La structure filonienne du Mourral de la Grave affleure sur toute la hauteur du versant surplombant la vallée de l'Orbiel. Elle est donc facile à attaquer directement à plusieurs niveaux. Des percements horizontaux en travers-banc sur les flancs du gisement supposent de longs percements, très couteux en temps, efforts et investissement. De plus, le fait d'attaquer directement les zones minéralisées permet d'augmenter le rapport rentabilité-production/effort-investissement. Cette volonté de rester efficace au moindre effort d'abattage, mais au détriment d'un certain confort pour la circulation et le portage, découlerait aussi du gabarit des galeries de recherche dépassant rarement $1 \mathrm{~m}$ de hauteur (figure 2d).

\section{Conclusions et PERSPECTIVES}

Bien que les recherches, menées entre 2009 et 2012, n'aient pas permis d'appréhender le complexe minier des Barrencs dans toute son extension, ses dimensions et sa complexité, elles le placent parmi les plus grandes mines protohistoriques et antiques de la France. À l'avenir, d'autres campagnes seront nécessaires pour mesurer sa véritable place dans l'économie de la Gaule méditerranéenne entre les IV $^{\mathrm{e}}$ et $\mathrm{I}^{\mathrm{er}}$ siècles av. n. è. Cependant, les recherches, concentrées notamment sur la partie centrale de la structure filonienne du Mourral de la Grave, ont permis de rassembler de nombreuses données sur l'architecture des ouvrages, sur les techniques et la dynamique d'exploitation, la chronologie relative et absolue (en stratigraphie) des travaux miniers, 
ainsi que sur la nature et la disposition spatiale des ressources exploitées par les Anciens. La grandeur, l'homogénéité morphologique et l'organisation rationnelle des ouvrages, la connaissance et la maîtrise des techniques minières prouvent, en dehors d'une mise en œuvre par un personnel qualifié et bien encadré, une mise en gestion à long terme de l'ensemble du complexe minier à travers d'imposants travaux de recherche et d'assistance creusés par plusieurs équipes (figure 2c). Ce fait est d'autant plus remarquable que les données chronologiques des travaux, essentiellement réalisés bien avant l'arrivée des Romains, placent l'exploitation principale entre le $\mathrm{IV}^{\mathrm{e}}$ et le $\mathrm{II}^{\mathrm{e}}$ siècle av. $\mathrm{n}$. è. Mais ce constat devrait moins nous surprendre aujourd'hui, car nous savons maintenant qu'au tournant du Iv ${ }^{\mathrm{e}}$ siècle av. n. è., les mines d'or en roche des Lémovices étaient intensivement exploitées dans la Gaule indépendante (Cauuet, 2004). Aux Barrencs, témoignant d'une très bonne connaissance empirique de la gîtologie du gisement, les mineurs se sont « laissés guider " par les filons et les failles à la recherche du minerai. Les indices issus de l'étude géologique laissent peu de doutes sur la nature de la production. La minéralisation d'origine épithermale abattue dans les chantiers anciens d'exploitation est constituée d'une association de sulfures et de sulfosels dans laquelle prédominent la pyrite, la chalcopyrite, la freibergite, la tétraédrite et la galène argentifère, la sphalérite et d'autres minéraux plus ou moins complexes de l'argent, du cuivre et du plomb. Le cuivre et le plomb peuvent présenter des teneurs élevées (plus de $10000 \mathrm{~g} / \mathrm{t}$ ) et l'argent peut atteindre aussi des valeurs importantes, parfois remarquables (plus de $1000 \mathrm{~g} / \mathrm{t}$ ), notamment dans les zones de croisement des panneaux filoniens principaux (F1, F2, F3), lieux privilégiés de l'extraction. Même si les indices dont nous disposons concernent le début de la chaîne opératoire pour la production des métaux, la présence importante et constante de ces trois métaux dans des associations minérales étroitement entrelacées (figures 5 e et $\mathrm{f}$ ) rend peu probable le fait qu'un de ces métaux ait pu être ignoré au cours du processus d'extraction métallurgique. Mais pour confirmer cette hypothèse, il faudrait pouvoir étudier des sites de traitement du minerai. Actuellement aucun établissement de ce type contemporain de la mine n'a encore été identifié sur le versant sud de la Montagne Noire. Il s'agit là d'une des perspectives de recherche à envisager, notamment près des ouvrages de surface, sur le plateau et le versant méridional du site des Barrencs.

Au-delà des questions soulevées, quant à son organisation et à son administration au niveau local, la mine des Barrencs ouvre des perspectives, jusqu'alors insoupçonnées, sur l'importance de l'activité minière au second âge du Fer dans la Gaule méridionale. A ce stade, la production des métaux régionaux doit être prise en considération avec l'ensemble des changements économiques qui se produisent, notamment au tournant des III $^{\mathrm{e}}$ et $\mathrm{II}^{\mathrm{e}}$ siècles av. n. è (Brun et Ruby, 2008). Cette période voit le renforcement de la puissance romaine en Méditerranée, vainqueur de la Seconde Guerre Punique, et l'intensification du commerce des produits italiques dans le Sud de la Gaule (Rancoule, 1985; Moret 2002; Gorgues 2010). Le III $^{\mathrm{e}}$ siècle av. n. è. correspond aussi à l'essor de la valeur-argent constaté depuis la Gaule méditerranéenne le long des Pyrénées et jusqu’au Nord-Est de la péninsule Ibérique (Mantenant, 2014), renforcé au $\mathrm{II}^{\mathrm{e}}$ siècle av. n. è. par l'intensification de la frappe de monnaies gauloises et ibériques (Callegarin et al., 2013). Dans quelle mesure cette évolution a-t-elle influencé l'exploitation des gisements du bassin de la Garonne à celui de l'Aude? Les données recueillies aux Barrencs (Beyrie et al., 2014; Mantenant, 2014), ainsi que par exemple celles issues du territoire des Rutènes (Léchelon, 2011), de la haute vallée de l'Orb (Gourdiole et Landes, 1998), du massif des Corbières (Mantenant, 2014; Mantenant et al., 2015) et des Hautes Pyrénées (Girard et al., 2010) constituent un début de réponse à développer.

\section{Remerciements}

Nous tenons à remercier particulièrement le Laboratoire GET (UMR 5563) - université Paul-Sabatier (Toulouse) pour l'accueil et le support technique et logistique accordés à la réalisation des analyses au MEB et à la microsonde électronique.

\section{Bibliographie}

Beyrie A., Fabre J.-M., Kammenthaler E., Mantenant J., Munteanu G., Rico C., 2011a. La mine antique de cuivre des Barrencs (Lastours et Fournes-Cabardes, Aude). L'Archéologue, $113: 50-53$.

Beyrie A., Fabre J.-M., Kammenthaler E., Mantenant J., Munteanu G., Rico C., 2011b. Une vaste exploitation minière du second âge du Fer. La mine de cuivre des Barrencs (Lastours, Fournes-Cabardès, Aude). Revue Archéologique de Narbonnaise, XLIII : 29-46.

Beyrie A., Fabre J.-M., Kammenthaler E., Mantenant J., Munteanu G., Rico C., 2014. La mine de cuivre antique des Barrencs (Lastours et Fournes-Cabardès, Aude), DFS, programme de recherche triennale 2010-2012. SRA LanguedocRoussillon, Montpellier, 113 p.

Brun P., Ruby P., 2008. L'áge du Fer en France. Premières villes, premiers états celtiques, Ed. La Découverte, Collection Archéologie de la France, Paris, 180 p. 
Callegarin L., Geneviève V., Hiriart E., 2013. Production et circulation monétaire dans le sud-ouest de la Gaule à l'âge du Fer (III $-\mathrm{I}^{\mathrm{er}}$ s. a.C.). Dans L'âge du Fer en Aquitaine et sur ses marges. Mobilité des hommes, diffusion des idées, circulation des biens dans l'espace européen à l'âge du Fer, Actes du $35^{\mathrm{e}}$ colloque de l'AFEAF, Bordeaux, 2-5 juin 2011, Aquitania Suppl. 30, Bordeaux, 185-218.

Caunet B., 2004. Lor des Celtes du Limousin. Culture et Patrimoine en Limousin, Limoges, 124 p.

Crouzet J., Tollon F., 1980. Le gisement stratiforme et filonien de Salsigne, Aude. Mémoires du BRGM, 112, Orléans : 54 p.

Decombeix P.-M. Fabre J.-M., Rico C., 2001. Mines et sidérurgie antiques sur le versant méridional de la Montagne Noire. Prospection thématique 2000-2002, rapport intermédiaire 2001. SRA Languedoc-Roussillon, Montpellier, 28 p.

Domergue C., 1993. Un centre sidérurgique romain de la Montagne Noire: Le domaine des Forges (Les Martys, Aude), suppl. 27 à la Revue Archéologique de Narbonnaise, Paris, 477 p.

Domergue C., 2008. Les mines antiques. La production des métaux aux époques grecque et romaine, Collection Antiqua, 11, A. et J. Picard, Paris, 280 p.

Esparseil M., 1893. Régime minéral du département de l'Aude. 1ère partie : minerais de fer. Bull. SESA, IV : 177-239.

Fabre J.-M., Beyrie A., Kammenthaler E., Mantenant J., Munteanu G., Rico C., 2012. La mine des Barrencs (Lastours, Fournes). Patrimoines, vallées des Cabardès, 7 : 23-28.

Fabre J.-M., Domergue Cl., Dabosi Fr., 2016. Le fer romain de la Montagne Noire. Martys 2 : les débuts. 25 années de recherches pluridisciplinaires (1988-2013), suppl. 43 à la Revue Archéologique de Narbonnaise, Montpellier, 560 p.

Galant P., 2001. Concession minière de Lastours (Aude). Rapport concernant l'évaluation de l'intérêt patrimonial du site dit des Barrencs de Fournes. DRAC-SRA Languedoc-Roussillon, Montpellier, p. 3.

Girard J., Munoz M., Cauuet B., Polve M., Aries S., Callegarin L., 2010. Mines d'argent du Montaigu (HautesPyrénées, France): une filière aquitaine de l'argent? Étude isotopique du plomb, Archéosciences, 34 : 235-241.

Gorgues A., 2010. Economie et société dans le Nord-Est du domaine

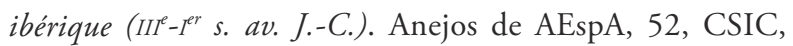
Madrid, $494 \mathrm{p}$.

Gourdiole R., Landes C., 1998. La Transalpine minière : des monts d'Orb au bassin de Lodève. In L. Schneider et D. Garcia (dir.). Carte archéologique de la Gaule. Le Lodévois (34/1).
Académie des Inscriptions et Belles-Lettres, Fondation Maison des Sciences de l'Homme, Paris, 53-66.

Issard H., 1984. Comparaison lithostratigraphique, géochimique et structurale entre la zone axiale et les nappes du versant Sud de la Montagne Noire dans le district aurifère de Salsigne (Aude, France). Thèse de $3^{\mathrm{e}}$ cycle, Université Saint-Étienne Jean-Monnet, France, 244-245.

LÉCHELON B., 2011. Argent rutène et entrepreneurs romains aux confins de la Transalpine. In P. Gruat, J.M. Pailler, D. Schaad (dir.). Les Rutènes: du peuple à la cité, de l'indépendance à l'installation dans le cadre romain 150 a.C.-100 p.C., colloque de Rodez et Millau (Aveyron), novembre 2007. Suppl. 25 d'Aquitania, Bordeaux, 245-279.

Moret P., 2002. Tolosa, capitale des Volques Tectosages. In J.-M. Pailler (dir.). Tolosa. Nouvelles recherches sur Toulouse et son territoire dans l'Antiquité. Collection de l'École Française de Rome, 281, Rome, 80-88.

Rancoule G., 1985. Observations sur la diffusion des importations italiques dans l'Aude aux $\mathrm{II}^{\mathrm{e}}$ et $\mathrm{I}^{\mathrm{er}}$ s. avant J.-C. Revue Archéologique de Narbonnaise, XVIII : 263-275.

Mantenant J., Beyrie A., Fabre J.-M., Kammenthaler E., Munteanu G., Rico C., 2013. Les Barrencs, une vaste mine protohistorique et antique en Montagne Noire. Bulletin de la Société d'Études Scientifiques de l'Aude, CXIII : 27-44.

Mantenant J., 2014. Montagnes métalliferes de Gaule méditerranéenne. Approche archéologique et historique de la production des métaux en Languedoc occidental du début du second âge du Fer à la fin de la période romaine (IV s. av. n. è.-Ves. de n. è.). Thèse de doctorat, Université Toulouse II - Le Mirail, France, 143-247, 301-319 et 375-382.

Mantenant J., Munoz M., 2015. La caractérisation d'une production d'argent en Languedoc occidental durant l'époque romaine républicaine ( $I^{e}-\mathrm{I}^{e r}$ s. av. n. è.), communication au $20^{\mathrm{e}}$ coll. GMPCA, avril 2015, Besançon.

Morin D., 2001. Les anciennes mines des Barrencs (communes de Fournes-Cabardès et de Lastours). Rapport d'expertises. DIREN Languedoc-Roussillon, Montpellier, 28 p.

TAMAs C., 2010. Endogenous breccia structures (brecia pipe and greccia dykes) and the petro-metallogeny of Rosia Montana ore deposit (Metaliferi Mountains, Romania). MEGA, Cluj-Napoca, $168 \mathrm{p}$.

Tollon F., 1970. Le district aurifère de Salsigne, Aude. Thèse d'État, Université Toulouse III - Paul Sabatier, France, 175 p.

Yueshi Lei, 1993. La tectonique cassante du gisement aurifere de Salsigne (Aude, France). Éditions BRGM, Orléans, 158 p. 


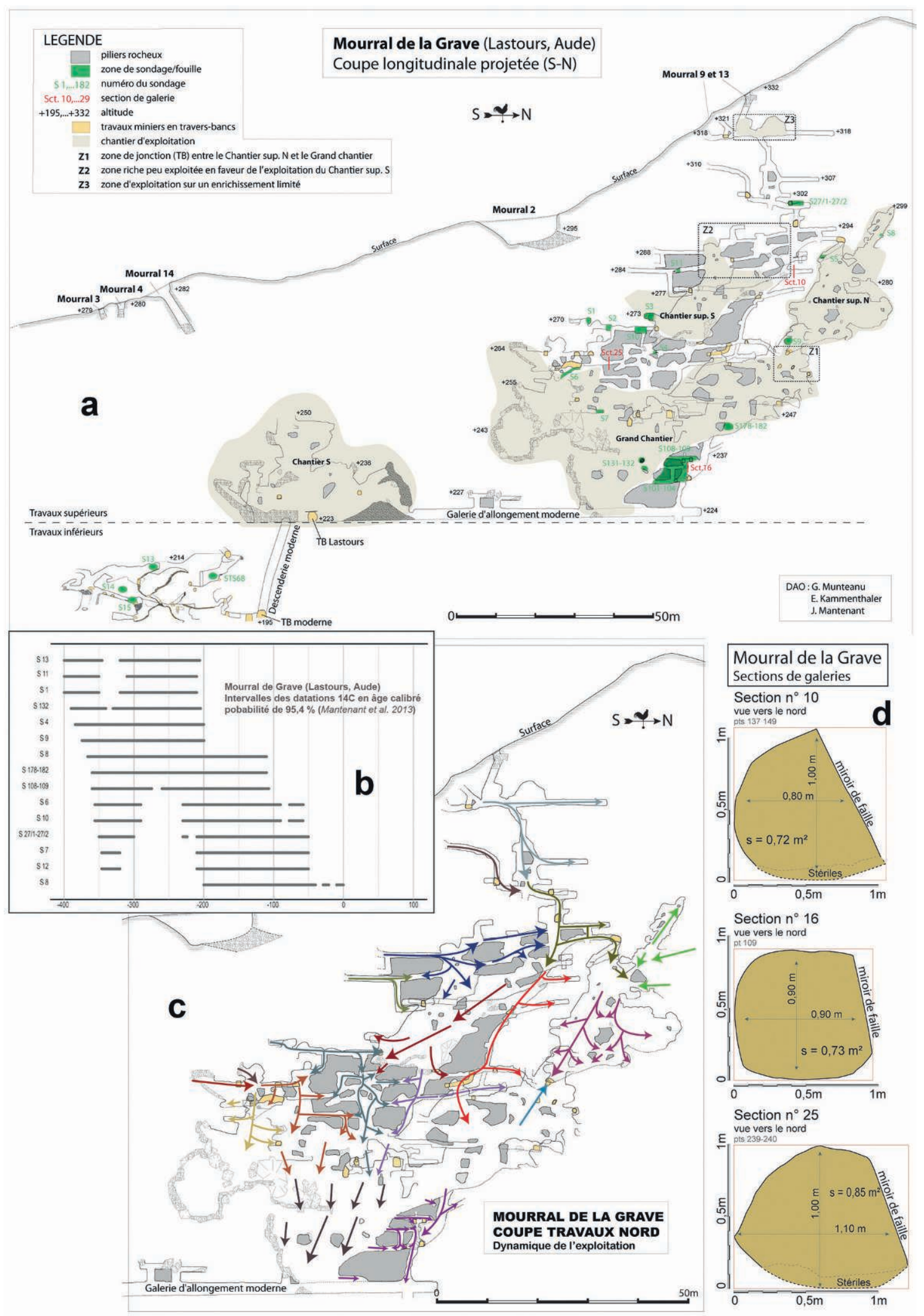

Figure 2 : Gabriel Munteanu et al., Le complexe minier gaulois des Barrencs (Aude, France) dans son contexte géologique et minéralogique (p. 167) 


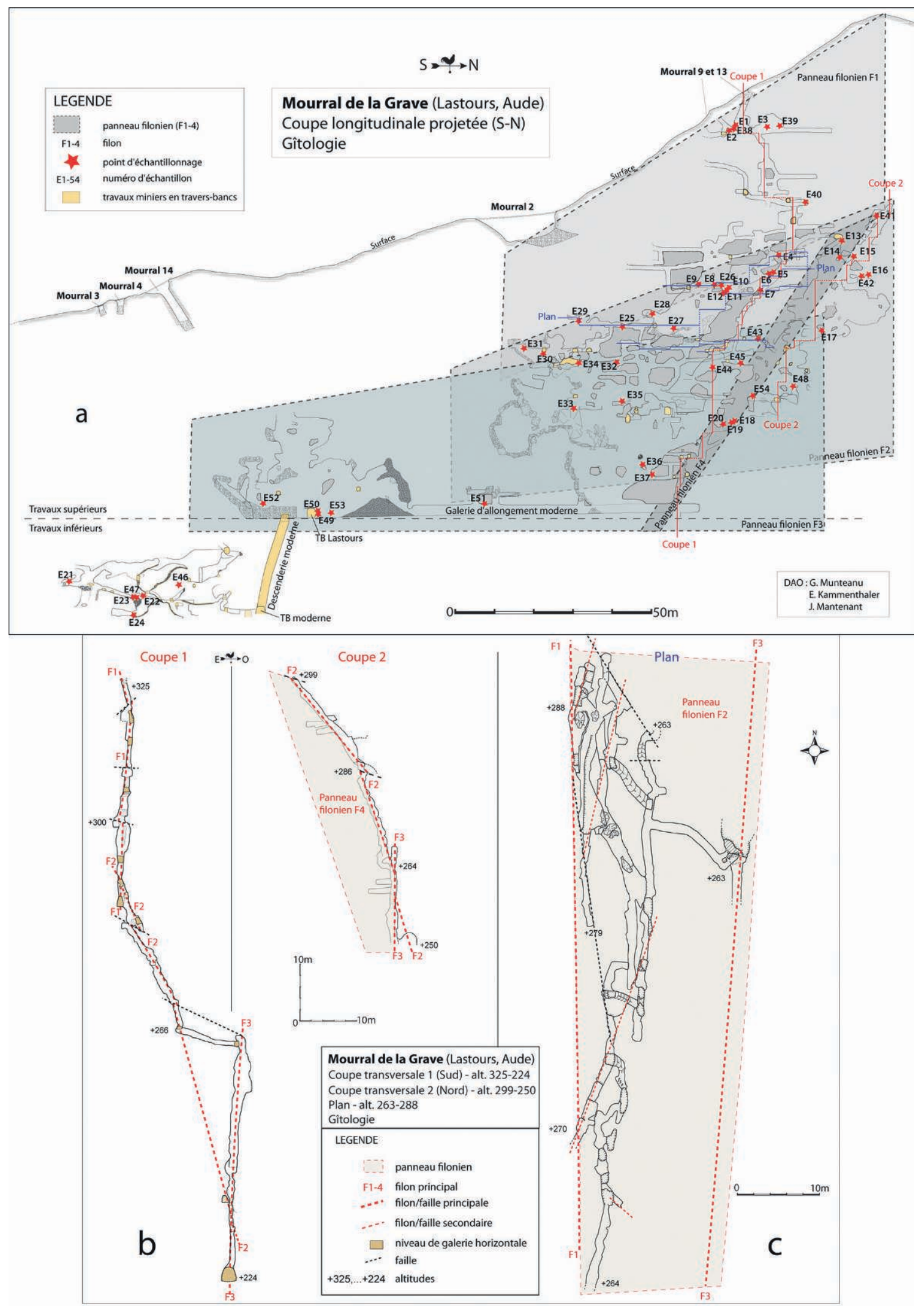

Figure 3 : Gabriel Munteanu et al., Le complexe minier gaulois des Barrencs (Aude, France) dans son contexte géologique et minéralogique (p. 169) 




Figure 4 : Gabriel Munteanu et al., Le complexe minier gaulois des Barrencs (Aude, France) dans son contexte géologique et minéralogique (p. 170) 


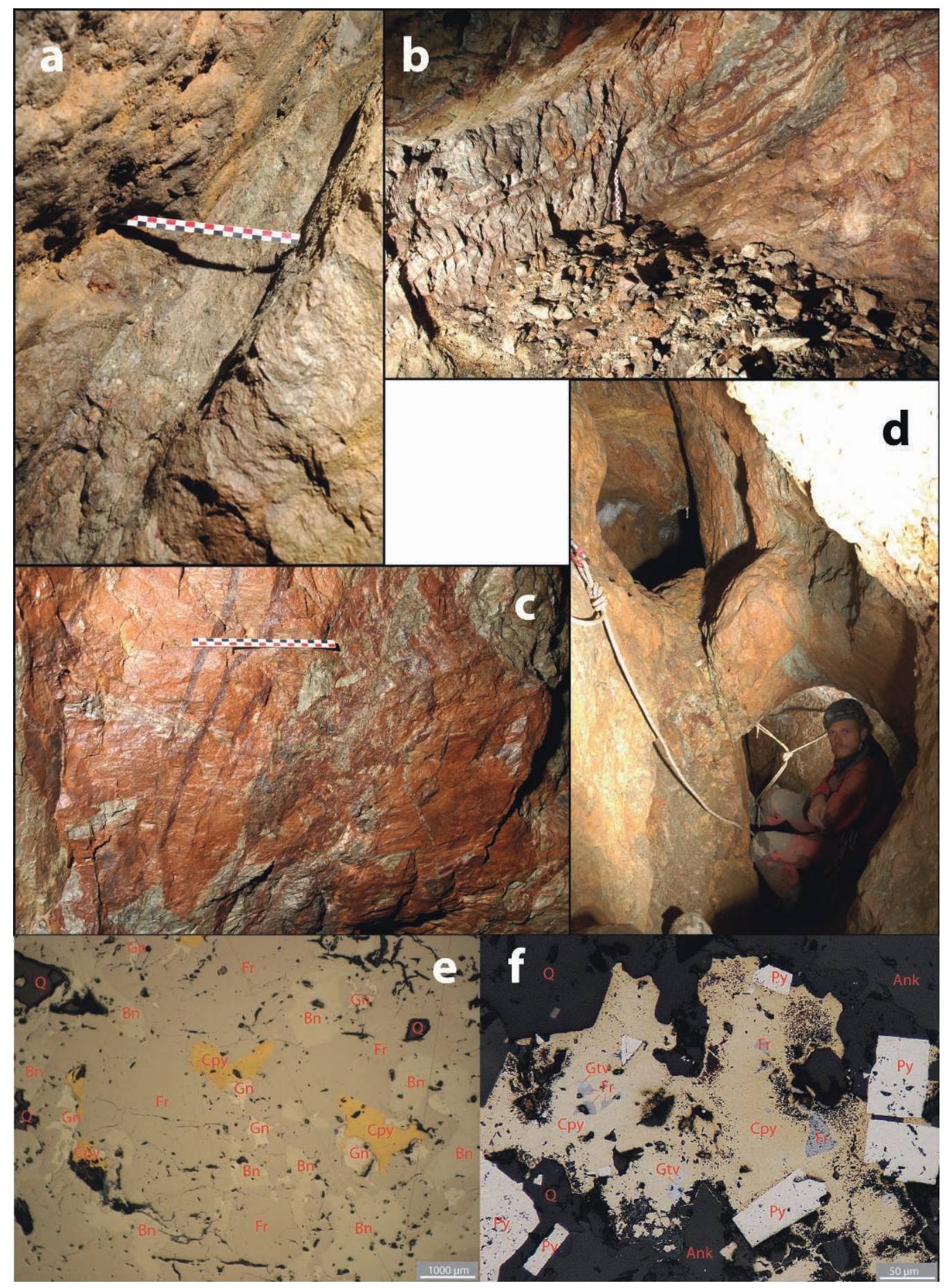

Figure 5 : Gabriel Munteanu et al., Le complexe minier gaulois des Barrencs (Aude, France) dans son contexte géologique et minéralogique (p. 171) 